\title{
SYNTAXONOMY AND SITE ECOLOGY OF A CENTRAL ITALY FOREST LANDSCAPE
}

\author{
Federico Maria TARDELLA ${ }^{1}$, Alessandra VITANZI ${ }^{2}$, \\ Daniele SPARVOLI ${ }^{2} \&$ Andrea CATORCI ${ }^{1}$
}

\begin{abstract}
A phytosociological survey of a woodland located in the central part of Umbria (Central Italy) was carried out using the Braun-Blanquet method: 80 relevés were classified through cluster analysis. Nine forest syntaxa were reported and three subassociations (Erico arboreae-Quercetum cerridis lathyretosum veneti, Aceri obtusati-Quercetum cerridis arbutetosum unedonis and Cyclamino hederifolii-Quercetum ilicis quercetosum cerridis) were typified. Topographic data (altitude, aspect, slope, morphology) and pedological data (soil pH, texture and depth) were collected and a synecological analysis of syntaxa was performed. In order to understand the relation between environmental factors and plant communities, a Canonical Correspondence Analysis was run. The results showed soil parameters ( $\mathrm{pH}$, texture and depth) and altitude as the main ecological factors explaining the distribution of plant communities in the study area. The combination of topographic factors (aspect, morphology and slope angle) influences, by contrast revealed the distribution of forest syntaxa within homogeneous geo-pedological and bioclimatic conditions.
\end{abstract}

Key words: forest vegetation, phytosociology, synecology, geomorphology, pedology.

\section{Izvleček}

Izvedli smo fitosociološko raziskavo gozdov v srednjem delu Umbrije (srednja Italija) z Braun-Blanquetovo metodo: 80 popisov smo uvrstili s klastrsko analizo. Obravnavamo devet gozdnih sintaksonov in opisali smo tri subasociacije (Erico arboreae-Quercetum cerridis lathyretosum veneti, Aceri obtusati-Quercetum cerridis arbutetosum unedonis in Cyclamino hederifolii-Quercetum ilicis quercetosum cerridis). Zbrali smo topografske (nadmosrska višina, ekspozicija, naklon, morfologija terena) in pedološke podatke (pH, tekstura in globina tal) in naredili sinekološko analizo sintaksonov. Za pojasnitev povezave med rastiščnimi dejavniki in rastlinskimi združbami smo naredili Canonical Correspondence Analysis. Rezultati kažejo, da so dejavniki tal (pH, tekstura in globina) in nadmorska višina glavni rastiščni dejavniki, ki pojasnjejo razširjenost rastlinskih združb v obravnavanem območju. Kombinacija topografskih dejavnikov (ekspozicija, morfologija reliefa in naklon) je odločilna za pojavljanje gozdnih sintaksonov v homogenih geopedoloških in bioklimatskih razmerah.

Ključne besede: gozdna vegetacija, fitosociologija, sinekologija, geomorfologija, pedologija.

\section{INTRODUCTION}

The forest landscape of Central Italy is almost well known from a phytosociological point of view. During the last decades, in fact, a great number of papers have been published about this issue, making it possible to define the syntaxonomical framework, especially for the high-rank syntaxa (Biondi et al. 2001, 2002a, 2003, Allegrezza et al. 2002, Blasi et al. 2004). Also at the association level, all the most important forest types are nowadays inserted into an organic synsystematical framework. Although the relation between the distribution of phytosociological types and ecological parameters (e.g. geomorphology, soil and bioclimate), using quantitative environ-

\footnotetext{
${ }^{1}$ School of Environmental Sciences, UNICAM University of Camerino, via Pontoni 5, I-62032 Camerino (MC); e-mail: andrea.catorci@unicam.it, dtfederico.tardella@unicam.it

${ }^{2}$ School of Advanced Studies - PhD Course in Environmental Sciences and Public Health, UNICAM University of Camerino, via Lili 55, I-62032 Camerino (MC); e-mail: alessandra.vitanzi@unicam.it, daniele.sparvoli@unicam.it
} 
mental field data, has been rarely investigated, this kind of knowledge is, however, the basis for advancing in the comprehension of the ecological processes involved at plant community and landscape scales and for achieving the definitive phytosociological characterization of the plant communities (Zuccarello et al. 1999). Moreover, an accurate synecological analysis might allow for a better understanding of the interrelations between the botanical resource and the present and past forest use, and might also help to define some predictive models aimed at floristic biodiversity conservation and at leading the forest management plans.

On the basis of such premises, the research aim was to define the phytosociological placement of the plant communities of a little known submediterranean forest landscape and characterize them from a synecological viewpoint by direct quantitative measurements of some environmental parameters.

\section{MATERIALS AND METHODS}

\subsection{STUdy AREA}

The study area is located in the central part of Umbria (Central Italy) (coordinates $43^{\circ} 05^{\prime}$ $42^{\circ} 44^{\prime} \mathrm{N}$; $12^{\circ} 25^{\prime}-12^{\circ} 36^{\prime} \mathrm{E}$ ), at altitudes ranging from 150 to $700 \mathrm{~m}$ a.s.l. and is characterized by three geomorphological units (Servizio Geologico d'Italia 1980):

- sandy-clayey low hills (maximum altitude 250-300 m), with low slope angles;

- marly and marly-calcareous hills (maximum altitude 400-450 m), with intermediate slope angles;

- marly-arenaceous high hills (maximum altitude $600-700 \mathrm{~m}$ ), characterized by an alternation of sandstone and marly layers, which gives rise to different geo-pedological soil contexts, depending on the layers' outcropping and attitude (Catorci et al. 1994, Giovagnotti et al. 2003).

According to Orsomando \& Catorci (2000) the study area belongs to the lower Mesotemperate bioclimatic belt; only the tops of the highest hills (above 550-600 $\mathrm{m}$ ) are located between the lower Mesotemperate and the upper Mesotemperate bioclimatic belt. The main features of the two bioclimatic belts are reported in Table 1 .
The plant landscape is characterized by evergreen sclerophyllous woods with a dominance of Quercus ilex subsp. ilex or by deciduous woods with a dominance of Quercus pubescens s.l., Quercus cerris or Quercus frainetto.

\subsection{DAtA GOLleGtion}

In the period 2005-2008, 62 phytosociological relevés were carried out using the Braun-Blanquet phytosociological method (Braun-Blanquet 1964, Géhu \& Rivas-Martínez 1981, Biondi et al. 2004). Furthermore, 18 relevés, taken from Catorci \& Orsomando $(1997,1998)$ and Biondi et al. (2001), were added.

The soil data collected in the phytosociological relevé sites, were obtained using a graduated pole for soil depth measurement, a pH-meter and an electromagnetic sieve for texture.

\subsection{DATA PROGESSING AND STATISTICAL ANALYSIS}

The phytosociological data set was submitted to multivariate analysis (Westhoff \& van der Maarel 1978). The phytosociological values were transformed according to Van der Maarel (1979), obtaining a matrix made up of 177 rows (floristic units) $\times 80$ columns (relevés), which was submitted to a numerical classification using the Complete link algorithm (Orloci 1978), based on euclidean distance.

For the syntaxonomical placement of the vegetation types, reference was made to European publications (Rivas-Martínez et al. 2001, 2002), papers revising Italian vegetation (Biondi et al. 2003, Blasi et al. 2004) and local phytosociological studies. The species nomenclature follows Conti et al. (2005) and, in some cases, Pignatti (1982) and Tutin et al. (1964-1980, 1993); the life forms and the chorotypes follow Pignatti $(1982,2005)$.

For each syntaxon, frequency distributions of environmental variables (altitude, aspect, slope angle, morphology, soil $\mathrm{pH}$, depth and sand percentage), expressed in quartiles and graphically represented using box-plots, were calculated to focus attention exclusively on the central values of the sample.

Canonical Correspondence Analysis (CCA) on two matrices, 80 relevés (classified into syntaxa by phytosociological analysis) $\times 133$ spe- 
cies (average \% cover values of Braun-Blanquet's scale) and 80 relevés $\times 7$ environmental parameters (altitude, aspect, slope angle, morphology, soil $\mathrm{pH}$, depth and sand percentage), was carried out in order to identify the variability in the floristic data set explained by the ecological factors taken into consideration and their relation with plant communities. For data elaboration, species whose frequency exceeds $3 \%$ were selected.

Cluster analysis, CCA and descriptive statistical analyses were performed using SYN-TAX 2000 (Podani 2001), PC-ORD 5.0 (McCune \& Grace 2002, McCune \& Mefford 2006) and SPSS 13.0 (SPSS Inc. 2005) softwares.

\section{RESULTS}

\subsection{Phytosociological analysis}

The multivariate analysis of the 80 phytosociological relevés (Figure 1) shows two main clusters, each of which represents a physiognomic-ecological type. I - Deciduous woodlands (Quercetalia pubescenti-petraeae) with a dominance of Quercus cerris (subcluster Ia) or Quercus frainetto (subcluster Ib). II - Evergreen sclerophyllous woodlands mixed with deciduous trees (Quercetalia ilicis) (subcluster IIa) and deciduous woodlands (Quercetalia pubescenti-petraeae) with a dominance of Quercus pubescens s.l. (subcluster IIb).

The above-mentioned subclusters are divided into groups which correspond to the syntaxonomical level of association, subassociation or variant.

Erico arboreae-Quercetum cerridis Arrigoni in Arrigoni, Mazzanti \& Riccieri 1990

typicum

(Group Ia ${ }^{\mathrm{Ia} 1}$; Table 2, relevés 1-12; holotypus relevé 3 of Table 2 in Arrigoni, Mazzanti \& Riccieri 1990 - corresponding to the typical subass.)

lathyretosum veneti subass. nova

(Group Ia ${ }^{\mathrm{Ia} 2}$; Table 2, relevés 13-15; holotypus relevé 13 of Table 2)

Woodland with a dominance of Quercus cerris, with rich evergreen sclerophyllous undergrowth, managed as coppice with standards.

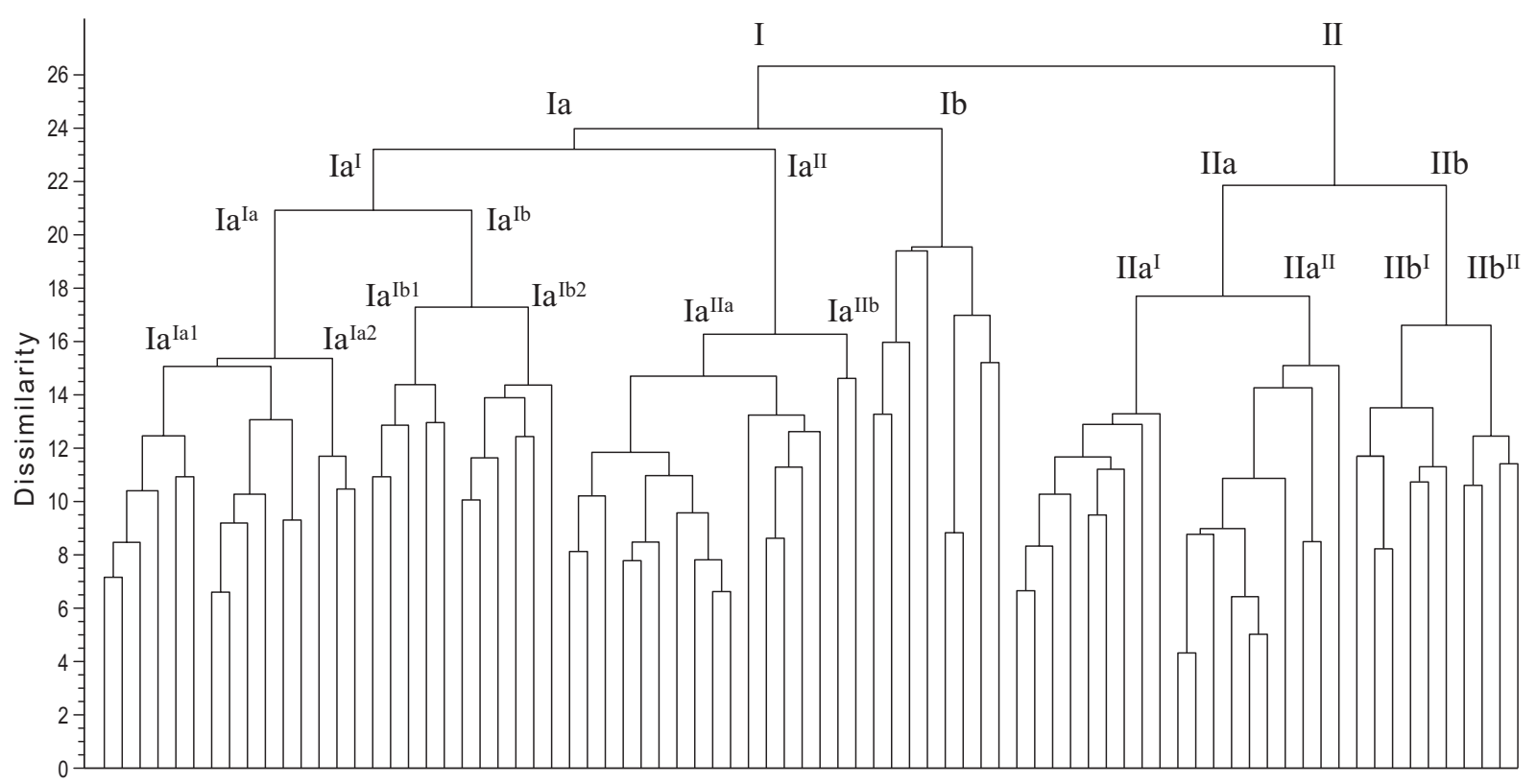

Figure 1: Dendrogram of phytosociological relevés.

Slika 1: Dendrogram fitosociološkihpopisov.

$\left(\mathrm{Ia}^{\mathrm{I} a 1}-\right.$ Erico arboreae-Quercetum cerridis typicum; $\mathrm{Ia}^{\mathrm{Ia} 2}-$ Erico arboreae-Quercetum cerridis lathyretosum veneti; $\mathrm{Ia}^{\mathrm{Ib}}-\mathrm{Ce}$ phalanthero longifoliae-Quercetum cerridis; $\mathrm{Ia}^{\mathrm{Ib} 2}$ - Cephalanthero longifoliae-Quercetum cerridis Castanea sativa variant; Ia ${ }^{\mathrm{II}}$ - Aceri obtusati-Quercetum cerridis arbutetosum unedonis; Ia ${ }^{\mathrm{Ilb}}$ - Aceri obtusati-Quercetum cerridis Carpinus betulus variant; $\mathrm{Ib}$ - Malo florentinae-Quercetum frainetto viburnetosum tini; $\mathrm{IIa}^{\mathrm{I}}$ - Cyclamino hederifolii-Quercetum ilicis quercetosum cerridis; $\mathrm{IIa}^{\mathrm{II}}$ - Cyclamino hederifolii-Quercetum ilicis cyclaminetosum hederifolii; $\mathrm{IIb}^{\mathrm{I}}$ - Roso sempervirentis-Quercetum pubescentis ericetosum multiflorae; $\mathrm{IIb}^{\mathrm{II}}$ - Roso sempervirentis-Quercetum pubescentis quercetosum pubescentis). 
From a phytosociological point of view, these woods are characterized by the dominance of Querco-Fagetea class species and by a fair number of transgressive elements from Quercetea ilicis class. Erico arboreae-Quercetum cerridis association was described by Arrigoni et al. (1990) for Tuscany and then reported by other authors (Biondi et al. 1995, Casini et al. 1995, Arrigoni 1997, Arrigoni \& Bartolini 1997, Arrigoni \& Di Tommaso 1997, Arrigoni et al. 1997, Scoppola 1998, Foggi et al. 2000, Biondi et al. 2002b) for many Italian districts.

The relevés $13-15$ of Table 2 differ from the other ones by the presence of some mesophilous species which identify a new subassociation named Erico arboreae-Quercetum cerridis lathyretosum veneti, differentiated by Lathyrus venetus, $\mathrm{Hi}$ eracium sylvaticum, Carpinus betulus, Melica uniflo$r a$ and Rosa arvensis. This new subassociation is spread in concave drainage surfaces (impluvia).

Cephalanthero longifoliae-Quercetum cerridis Scoppola \& Filesi 1998

(Group Ia ${ }^{\mathrm{Ib}}$; Table 3; holotypus relevé 5 of Table 1 in Scoppola \& Filesi 1998)

Castanea sativa variant

(Group Ia ${ }^{\mathrm{Ib} 2}$; Table 3, relevés 6-11)

Woodland with a dominance of Quercus cerris and Carpinus betulus, sometimes with the presence of Castanea sativa, managed as coppice with standards.

From a phytosociological point of view, these woods are characterized by the dominance of species belonging to Quercetalia pubescenti-petraeae order, with a fair number of elements of Fagetalia sylvaticae order, while the transgressive entities of Quercetea ilicis class are almost completely absent. Cephalanthero longifoliae-Quercetum cerridis was described by Scoppola \& Filesi (1998) for Monte Rufeno and Selva di Meana, between Latium and Umbria and identified by Biondi et al. (2002b) in Terni Province (Monte Peglia, Dorsale Amerina, Selva di Meana) and Di Pietro et al. (2010) on Monte Urbano and Monte Madonna, in the Tolfa-Ceriti Mountains (Northern Latium). In Central Italy several syntaxa of semimesophilous Quercus cerris woods were described (e.g. Arrigoni et al. 1990, Scoppola \& Filesi 1998, Catorci \& Orsomando 2001, Biondi et al. 2002b, Di Pietro et al. 2010). In particular, Cephalanthero longifoliaeQuercetum cerridis and Melico uniflorae-Quercetum cerridis seem to be very similar from an ecologic and floristic viewpoint. Despite the presence in the relevés of the study area, as well as in those of Terni (Biondi et al. 2002b) and Monte Rufeno (Scoppola \& Filesi 1998), of the characteristic species of the Melico uniflorae-Quercetum cerridis, which grows on both Flysch and volcanic substratum, the presence of the Cephalanthero longifoliae-Quercetum cerridis characteristic species set - which is absent, by contrast in Melico unifloraeQuercetum cerridis (Table 4) - and the different geopedological features, lead us to place the relevés of Table 3 into such syntaxon. This is also consistent with the conclusions of Biondi et al. (2002b) about Western Umbria Quercus cerris woods.

In the study area a Castanea sativa variant (relevés 6-11, Table 3) was identified. It represents the residual aspect of old cultivated fruitbearing chestnut groves, currently coppice managed, where an evolution process is going on towards the floristic-structural composition of Quercus cerris woods.

Aceri obtusati-Quercetum cerridis Ubaldi \& Speranza ex Ubaldi 1995

arbutetosum unedonis subass. nova

(Group Ia ${ }^{\text {IIa. }}$ Table 5, relevés 1-15; holotypus relevé 9 of Table 5)

Carpinus betulus variant

(Group Ia ${ }^{\mathrm{IIb}}$; Table 5, relevés 16-17)

Woodland with a dominance of Quercus cerris, with Ostrya carpinifolia and Acer opalus subsp. obtusatum, managed as mixed coppice.

From a phytosociological point of view, these woods are characterized by the dominance of Quercetalia pubescenti-petraeae order species, with a significant presence of transgressive elements from Quercetea ilicis class. Aceri obtusati-Quercetum cerridis association was described by Ubaldi \& Speranza (1982) for the Northern Marches and reported by other authors (Catorci \& Orsomando 2001, Allegrezza 2003, Taffetani et al. 2004, Argenti et al. 2006, Catorci et al. 2008) for Northern and Umbria-Marches Apennines (Central Italy).

In comparison with the Aceri obtusati-Quercetum cerridis typicum, the relevés collected in the study area are characterized by a higher presence of transgressive species from Quercetea ilicis class. Ubaldi (1988) describes the Aceri obtusati-Quercetum cerridis aceretosum monspessulani subassociation which also shows a group of Mediterranean species, among which, however, Arbutus unedo is absent. Such floristic difference and the consideration that Aceri obtusati-Quercetum cerridis aceretosum monspessulani develops on a carbonatic substratum, therefore in a neutral-basic soil con- 
text, while the woodlands present in the study area develop on marly-arenaceous rocks, so in a subacid soil context, allows us to define a new subassociation called Aceri obtusati-Quercetum cerridis arbutetosum unedonis, which is differentiated by Arbutus unedo and Rosa sempervirens.

In the study area a mesophilous aspect with Carpinus betulus (relevés 16-17 of Table 5) was also identified. It spreads on little alluvial terraces and small concave drainage surfaces (impluvia) and is characterized by the presence of a mesophilous group of species (Carpinus betulus, Pulmonaria apennina, Corylus avellana and Ulmus minor subsp. minor). Although this characterization highlights the existence of an ecological space for the definition of a new subassociation, we consider it is not appropriate to do so in this article, because of the low number of relevés. Thus, we insert this kind of forest community in a Carpinus betulus variant of Aceri obtusati-Quercetum cerridis.

Malo florentinae-Quercetum frainetto Biondi, Gigante, Pignattelli \& Venanzoni 2001

viburnetosum tini Biondi, Gigante, Pignattelli \& Venanzoni 2001

(Group Ib; Table 6; holotypus relevé 4 of Table 2 in Biondi, Gigante, Pignattelli \& Venanzoni 2001)

Woodland with a dominance of Quercus frainetto with Quercus cerris, managed as high forest. From a phytosociological point of view, these woods are characterized by the presence of Querco-Fagetea class species and of transgressive elements from Quercetea ilicis class (Catorci \& Orsomando 1998). Malo florentinae-Quercetum frainetto association was described by Biondi et al. (2001) for Central Italy (Umbria).

Cyclamino hederifolii-Quercetum ilicis Biondi, Casavecchia \& Gigante 2003

quercetosum cerridis subass. nova

(Group IIa'; Table 7, relevés 1-9; holotypus relevé 5 of Table 7)

cyclaminetosum hederifolii Biondi, Casavecchia \& Gigante 2003

(Group IIa ${ }^{\mathrm{II}}$; Table 7, relevés 10-19; holotypus relevé 2, Table 3 in Biondi, Casavecchia \& Gigante 2003)

Woodland with a dominance of Quercus ilex subsp. ilex, with Quercus pubescens s.l., Q. cerris and Fraxinus ornus subsp. ornus, managed as coppice with standards and, sporadically, as high forest.

From a phytosociological point of view, these woods are characterized by the dominance of
Quercetalia ilicis order and Quercetea ilicis class species and for the presence of a large number of transgressive species from Querco-Fagetea class. Cyclamino hederifolii-Quercetum ilicis association was described by Biondi et al. (2003), who report its presence in Liguria, Tuscany, Latium, Umbria, Marches, Apulia and Calabria, and also reported by other authors (Allegrezza et al. 2006, Foggi et al. 2006, Carranza et al. 2008, Tardella et al. 2010).

Two groups of Quercus ilex dominated woods were identified by cluster analysis. The first one ( $\mathrm{IIa}^{\mathrm{I}}$ ) is characterized by Quercus cerris and other acidophilous species, such as Asplenium onopteris, Calluna vulgaris, Erica arborea, Cytisus scoparius subsp. scoparius and Cistus salviifolius. This floristic characterization leads us to the definition of a new subassociation named Cyclamino hederifoliiQuercetum ilicis quercetosum cerridis differentiated by Quercus cerris, Erica arborea and Asplenium onopteris.

In comparison with Arbuto unedonis-Quercetum ilicis (Di Pietro et al. 2010), growing on the trachytic volcanic substratum of the Ceriti Mountains (Northern Latium), the floristic composition of Cyclamino hederifolii-Quercetum ilicis quercetosum cerridis is characterized by a higher number of elements from Querco-Fagetea class, as shown in the synoptic table (Table 8 ). Thus, this plant community may be considered as the subacidophilous ecological vicariant on arenaceous substrata, in the inner part of Central Italy, of the typical subassociation Cyclamino hederifolii-Quercetum ilicis cyclaminetosum hederifolii (Biondi et al. 2003), with which it shares several mesophilous elements. These features highlight that probably there is the ecological space for the definition of a new association of holm-oak wood growing on sandstones in Central Italy. However, it is not appropriate to do so in this article. Cyclamino hederifolii-Quercetum ilicis quercetosum cerridis, in fact, might be raised to this category only by extending the phytosociological research to a wider geographic area.

The second group ( $\mathrm{IIa}^{\mathrm{II}}$ ), by contrast, where the above mentioned acidophilous species are almost absent, can be attributed to Cyclamino hederifolii-Quercetum ilicis cyclaminetosum hederifolii.

Roso sempervirentis-Quercetum pubescentis Biondi 1986

ericetosum multiflorae Catorci \& Orsomando 1997 (Group IIb'; Table 9, relevés 1-6; holotypus relevé 2 of Table 2 in Catorci \& Orsomando 1997) 
quercetosum pubescentis Allegrezza, Baldoni, Biondi, Taffetani \& Zuccarello 2002

(Group IIb ${ }^{\mathrm{II}}$; Table 9, relevés 7-10; holotypus relevé 189 of Table 3 in Allegrezza et al. 2002, corresponding to relevé 4 of Table 18 in Biondi 1986)

Woodland with a dominance of Quercus pubescens s.l., often with Pinus halepensis, with undergrowth rich in evergreen sclerophyllous species, mainly managed as mixed coppice.

From a phytosociological point of view, these woods are characterized by the dominance of species belonging to Quercetalia pubescenti-petraeae order and by the presence of a significant group of transgressive species from Quercetalia ilicis order. Roso sempervirentis-Quercetum pubescentis association was described by Biondi (1986) for the sub-coastal area of the Marches and reported by other authors for Central Italy, also with reference to the various subassociations described (Biondi et al. 1990, 1992, 2002b, Pirone 1992, Casini et al. 1995, Biondi \& Allegrezza 1996, Allegrezza et al. 1997, 2002, Arrigoni \& Bartolini 1997, Arrigoni et al. 1997, Catorci \& Orsomando 1997, Blasi \& Di Pietro 1998, Scoppola 1998, Casini \& De Dominicis 1999, Foggi et al. 2000, Taffetani 2000, Catorci et al. 2008). In the study area the ericetosum multiflorae subassociation, described by Catorci \& Orsomando (1997), as well as the typical one, were identified.

\subsection{SYNECOLOGICAL DESGRIPTION}

The field data (altitude, geology, morphology, aspect, slope angle, soil depth, $\mathrm{pH}$ and sand \%) are shown in Table 10. The box-plot diagrams of the environmental factors are reported in Figure 2. The synecological characteristics of syntaxa are briefly described below.

Erico arboreae-Quercetum cerridis typicum - Syntaxon spread on South-facing slopes (SE-WSW), on North-facing watersheds (from NNW to ENE), with light slope angle (interquartile range $5-10^{\circ}$ ) and on flattened tops of reliefes. It develops on loamy sand subacid soils ( $\mathrm{pH}$ interquartile range 6.1-6.4), less deep than $50 \mathrm{~cm}$.

Erico arboreae-Quercetum cerridis lathyretosum venet - Subassociation differing from the typical one for developing in South-facing impluvia (concave drainage surfaces), on tendentially deeper soils.

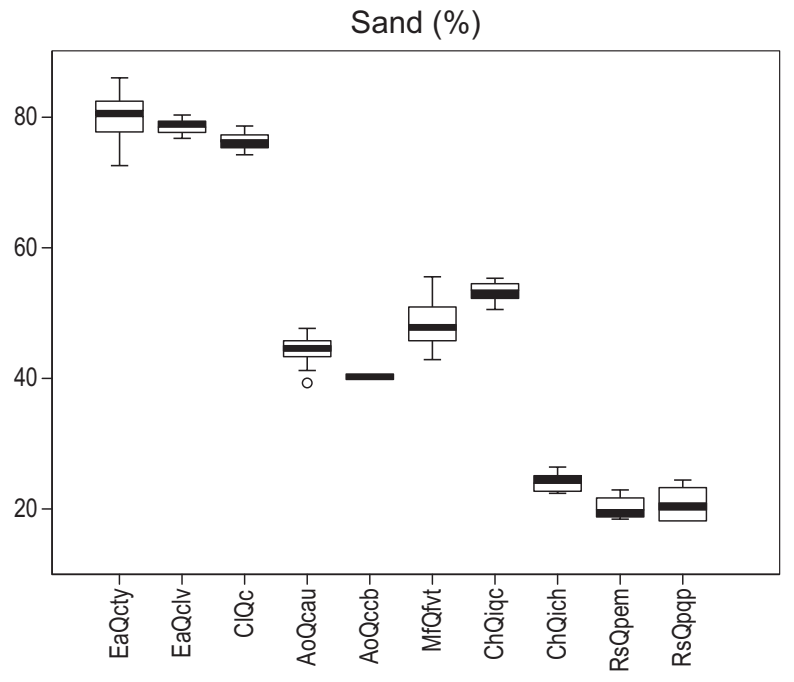

Figure 2: Box-plot diagrams of the environmental variables referred to the surveyed syntaxa.

(EaQcty - Erico arboreae-Quercetum cerridis typicum; EaQclv - Erico arboreae-Quercetum cerridis lathyretosum veneti; ClQc - Cephalanthero longifoliae-Quercetum cerridis; AoQcau - Aceri obtusati-Quercetum cerridis arbutetosum unedonis; AoQccb - Aceri obtusati-Quercetum cerridis Carpinus betulus variant; MfQfvt - Malo florentinae-Quercetum frainetto viburnetosum tini; ChQiqc - Cyclamino hederifolii-Quercetum ilicis quercetosum cerridis; ChQich - Cyclamino hederifolii-Quercetum ilicis cyclaminetosum hederifolii; RsQpem - Roso sempervirentis-Quercetum pubescentis ericetosum multiflorae; RsQpqp - Roso sempervirentis-Quercetum pubescentis quercetosum pubescentis). Aspect degrees are counted beginning from WNW so that Northern exposures range from $0^{\circ}$ (WNW) to $180^{\circ}$ (ESE), while Southern ones range from $181^{\circ}$ (ESE) to $360^{\circ}$ (WNW). Morphology (concavity/convexity) was quantified evaluating in each relevé site the angle formed by the tangent lines to the topographic surface on the horizontal plain.

Slika 2: Grafi (škatla z brki) rastiščnih spremenljivk obravnvanih sintaksonov.

(EaQcty - Erico arboreae-Quercetum cerridis typicum; EaQclv - Erico arboreae-Quercetum cerridis lathyretosum veneti; ClQc - Cephalanthero longifoliae-Quercetum cerridis; AoQcau - Aceri obtusati-Quercetum cerridis arbutetosum unedonis; AoQccb - Aceri obtusati-Quercetum cerridis Carpinus betulus variant; MfQfvt - Malo florentinae-Quercetum frainetto viburnetosum tini; ChQiqc - Cyclamino hederifolii-Quercetum ilicis quercetosum cerridis; ChQich - Cyclamino hederifolii-Quercetum ilicis cyclaminetosum hederifolii; RsQpem - Roso sempervirentis-Quercetum pubescentis ericetosum multiflorae; RsQpqp - Roso sempervirentis-Quercetum pubescentis quercetosum pubescentis). Ekspozicija je prikazana s stopinjami in se začne $\mathrm{z}$ WNW tako, da so severne ekspozicije od $0^{\circ}(\mathrm{WNW})$ do $180^{\circ}$ (ESE), južne pa od $181^{\circ}$ (ESE) do $360^{\circ}$ (WNW). Morfologijo reliefa (konkavnost/ konveksnost) smo opisali za vsak popis kot kot, ki ga tvorita tangenta na površino in horizont. 

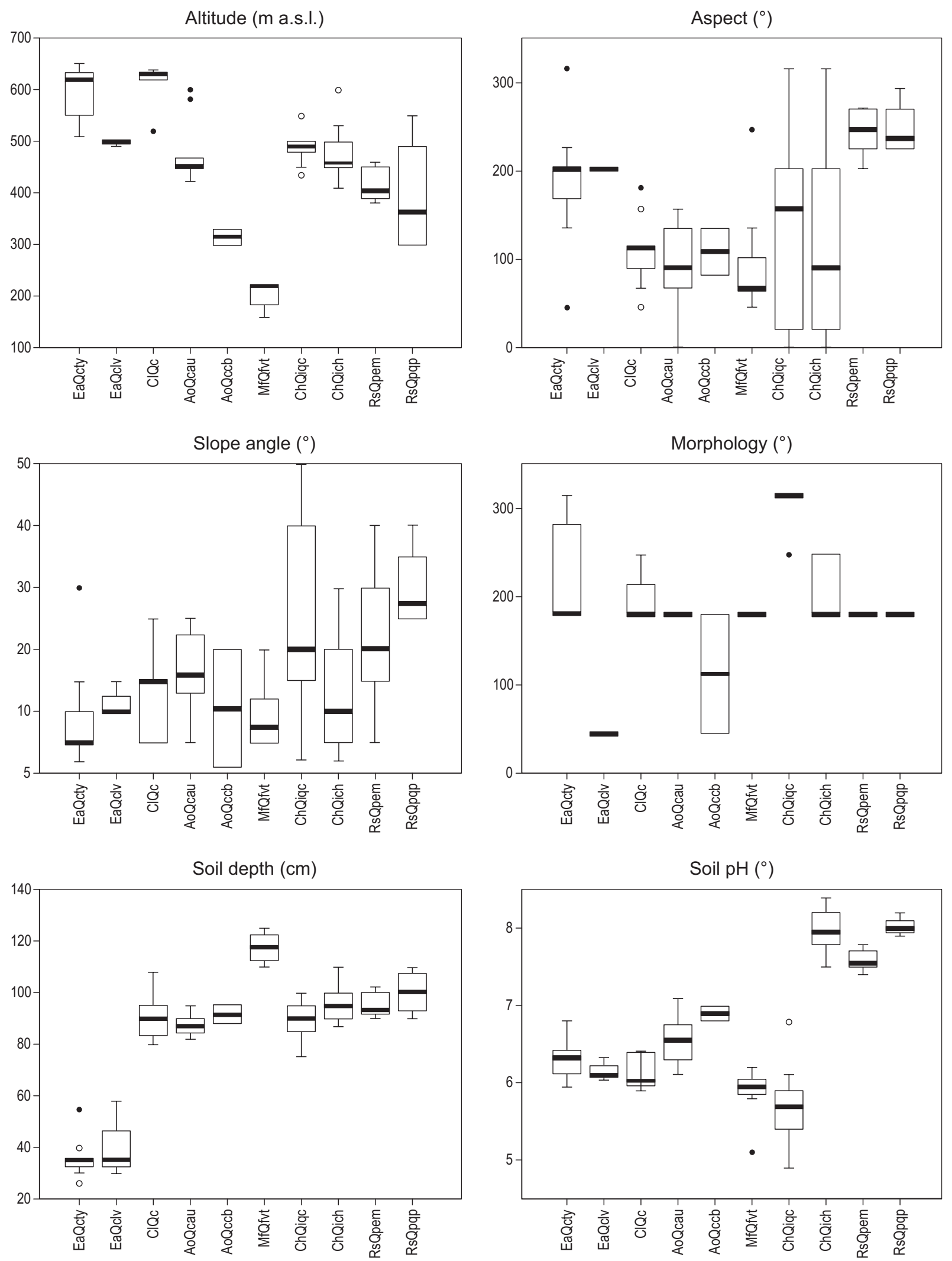

Figure 2 
Cephalanthero longifoliae-Quercetum cerridis Syntaxon spread mainly on North-facing (NNWE) lightly steep slopes (interquartile range $5-15^{\circ}$ ), on loamy sand subacid soils ( $\mathrm{pH}$ interquartile range 6.0-6.4), about $80-100 \mathrm{~cm}$ deep.

Aceri obtusati-Quercetum cerridis arbutetosum unedonis - Syntaxon spread on North-facing (WNW- E), lightly to moderately steep slopes (interquartile range $13-25^{\circ}$ ), on loamy subacid/ neutral soils ( $\mathrm{pH}$ interquartile range 6.2-6.8), about $80-100 \mathrm{~cm}$ deep.

Aceri obtusati-Quercetum cerridis Carpinus betulus variant - Syntaxon spread on little alluvial terraces of minor watercourses and on lightly to moderately steep $\left(15-20^{\circ}\right)$ marly colluvial deposits within small concave drainage surfaces (impluvia). It develops on clay loam/loam neutral soils (pH interquartile range $6.8-7.0$ ), about $80-100$ $\mathrm{cm}$ deep.

Malo florentinae-Quercetum frainetto viburnetosum tini - Syntaxon spread on flat valley bottoms or lightly steep slopes $\left(5-13^{\circ}\right)$, on loamy acid soils ( $\mathrm{pH}$ interquartile range 5.8-6.1), deeper than $100 \mathrm{~cm}$.

Cyclamino hederifolii-Quercetum ilicis cyclaminetosum hederifolii - Syntaxon spread mainly on North-facing (WNW-ENE), lightly to moderately steep slopes (interquartile range is about $10-20^{\circ}$ ) and on flat and semi-flat morphologies on the reliefe tops. It develops on silty loam or silty clay loam subalkaline soils $(\mathrm{pH}$ interquartile range 7.8-8.2), about 85-110 cm deep.

Cyclamino hederifolii-Quercetum ilicis quercetosum cerridis - Syntaxon spread on North-facing (WNW-E) or South-facing (SE-WSW), lightly to very steep watersheds (interquartile range 15$45^{\circ}$ ) and on relief tops. It develops on sandy clay loam acid soils ( $\mathrm{pH}$ interquartile range 5.4-6.0), about $80-100 \mathrm{~cm}$ deep.

Roso sempervirentis-Quercetum pubescentis quercetosum pubescentis - Syntaxon spread on moderately to very steep South-facing (SSE-SW) slopes (interquartile range $25-38^{\circ}$ ), on silty loam subalkaline soils $(\mathrm{pH}$ interquartile range 7.9-8.2), about $90-110 \mathrm{~cm}$ deep.

Roso sempervirentis-Quercetum pubescentis ericetosum multiflorae - Syntaxon spread on moderately to very steep South-facing (SE-SSW) slopes (interquartile range $13-33^{\circ}$ ), on silty loam subalkaline soils ( $\mathrm{pH}$ interquartile range 7.5-7.7), about $90-100 \mathrm{~cm}$ deep.

Canonical Correspondence Analysis results (Table 11) highlight that axis 1, explaining 11.5
$\%$ of variance in the species data set, is positively correlated to soil sand \% (0.751) and negatively to soil $\mathrm{pH}(-0.779)$. Axis 2, explaining $7.6 \%$ of variance, is positively related to altitude (0.968) and negatively to soil depth (-0.698); axis 3 , explaining the $4.2 \%$ of variance, is linked to aspect (0.751). As shown in Figure 3, CCA reveals the highest correlation between axis 1 and Cephalanthero longifoliae-Quercetum cerridis (positive), and with Roso sempervirentis-Quercetum pubescentis ericetosum multiflorae, Roso sempervirentis-Quercetum pubescentis quercetosum pubescentis and Cyclamino hederifolii-Quercetum ilicis cyclaminetosum hederifolii (negative). Axis 2 shows a negative relation particularly with Malo florentinae-Quercetum frainetto viburnetosum tini and Aceri obtusati-Quercetum cerridis Carpinus betulus variant and a positive relation mainly with Erico arboreae-Quercetum cerridis typicum.

\section{DISCUSSION}

The research results demonstrate that the forest syntaxa distribution is related, first of all, to the different pedological conditions, which, in turn, are determined by the kind of bedrock (Cremaschi \& Rodolfi 1991). Furthermore, within each geological group belonging to a bioclimatic belt, the combination of topographic factors is responsible for a further diversification of the forest landscape, also because aspect, morphology and slope angle have a feedback effect on pedological features, influencing some soil characteristics, such as soil depth (Pieruccini 2007, Agnelli et al. 2008).

Synecological analysis (Figure 2, Table 10) highlights that each syntaxon presents a peculiar set of environmental attributes which supported the phytosociological interpretations. Canonical Correspondence Analysis (Figure 3, Table 11) indicated soil parameters ( $\mathrm{pH}$, texture, depth) and altitude, as the main driving forces of floristic differentiation in the study area. The combination of topographic factors (aspect, morphology and slope angle), acting at a lower hierarchical level, seems to determine the distribution of forest syntaxa within homogeneous geo-pedological and bioclimatic conditions.

Table 12 presents schematically the hierarchical model of the considered combination of ecological factors which determine the diversity of the studied forest landscape. In particular, 


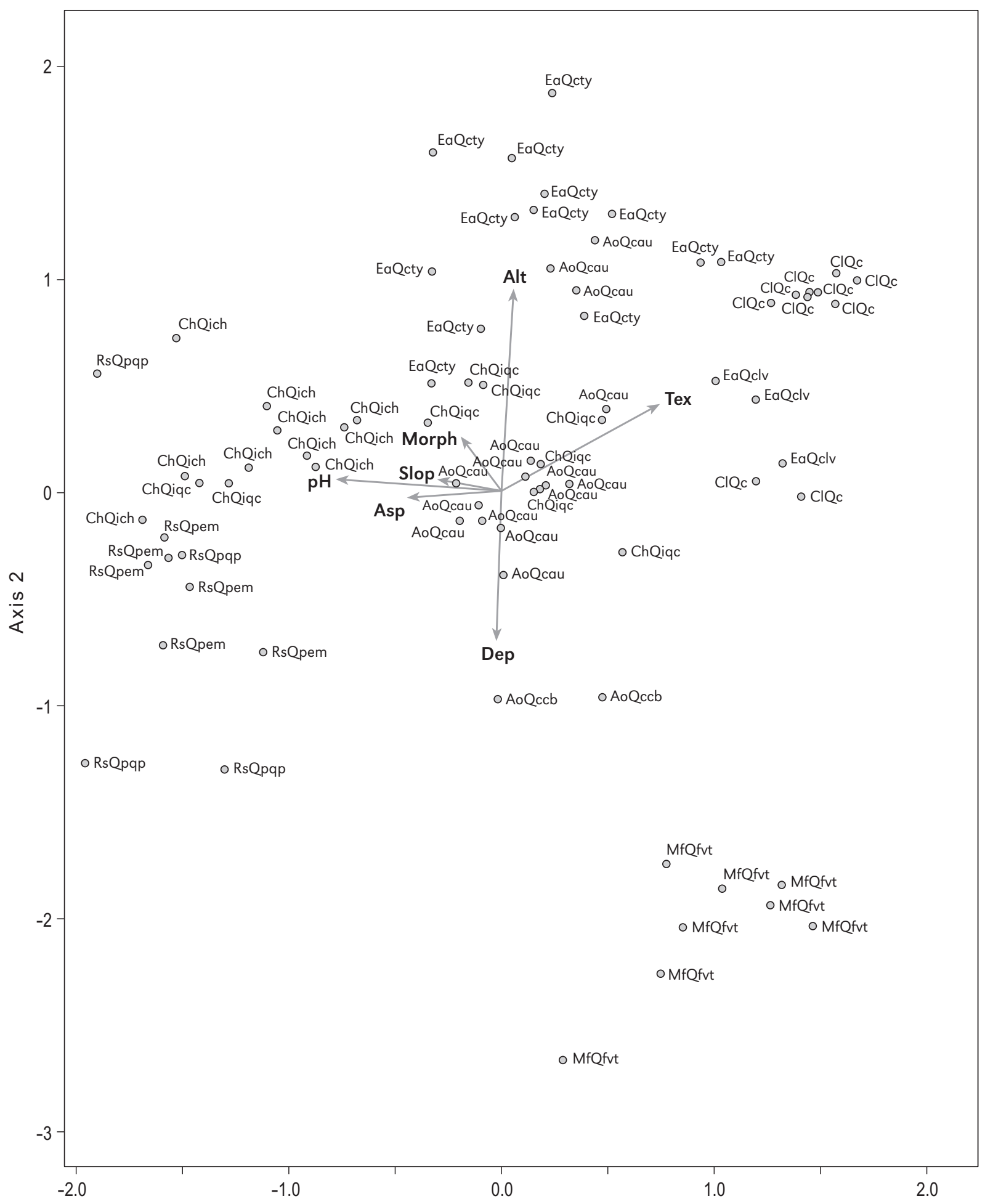

Figure 3: Canonical Correspondence Analysis joint plot. Only the relevés sites, corresponding to the forest syntaxa classified by phytosociological analysis, are reported. Labels are the same used in Figure 2.

Figure 3: Graf kanonične korespondenčne analize. Prikazani so samo popisi gozdnih sintaksonov, ki smo jih uvrstili s fitosociološko analizo. Oznake so iste kot pri Sliki 2. 
Roso sempervirentis-Quercetum pubescentis ericetosum multiflorae, Roso sempervirentis-Quercetum pubescentis quercetosum pubescentis and Cyclamino hederifolii-Quercetum ilicis cyclaminetosum hederifolii share some pedological features (subalkaline, moderately deep soils, with very low sand content) which differentiate them from the other syntaxa, growing on neutral and acid soils, which show, in turn, a separation at a lower hierarchical level, into two subgroups with different soil textures: Erico arboreae-Quercetum cerridis typicum, Erico arboreae-Quercetum cerridis lathyretosum veneti, Cephalanthero longifoliae-Quercetum cerridis and Cyclamino hederifolii-Quercetum ilicis quercetosum cerridis, growing on mainly sandy soils; Malo florentinae-Quercetum frainetto viburnetosum tini, Aceri obtusati-Quercetum cerridis arbutetosum unedonis and Aceri obtusati-Quercetum cerridis Carpinus betulus variant, growing on loam or clay loam soils. In each group, the relevés clusters are further differentiated by altitude, soil depth and the combination of topographic factors (aspect, morphology and slope angle) which contribute to determining the site dryness/mesophily level.

\section{CONCLUSION}

The characterization of pedologic and topographic parameters, related to the nine forest syntaxa pointed out by the research, proved to be useful to allow for a more certain phytosociological placement of the different clusters emerging from the multivariate analysis of the phytosociological relevés. Furthermore, the collection of the above mentioned parameters allows for the elaboration of predictive models concerning the distribution of the different vegetation series, permits an $e x$ post control of the phytosociological interpretations and the comparison of the existing forest vegetation with the expected one. The detection of deviations could move the research toward an understanding of the causes of such differences, for instance local geomorphological peculiarities, land use history (Decocq 2000) or presence of soils derived from ancient pedogenetic events (Catorci et al. 1993).

\section{Syntaxonomic scheme}

Quercetea ilicis Br.-Bl. ex A. \& O. Bolòs 1950

Quercetalia ilicis Br.-Bl. ex Molinier 1934 em. Riv.-Mart. 1975

Fraxino orni-Quercion ilicis Biondi, Casavecchia \& Gigante 2003

Cyclamino hederifolii-Quercetum ilicis Bion-

di, Casavecchia \& Gigante 2003

cyclaminetosum hederifolii Biondi, Casavecchia \& Gigante 2003

quercetosum cerridis subass. nova

Querco-Fagetea Br.-Bl. \& Vlieger in Vlieger 1937

Quercetalia pubescenti-petraeae Klika 1933 corr.

Moravec in Beguin \& Theurillat 1984

Carpinion orientalis Horvat 1958

Laburno anagyroidis-Ostryenion carpinifo-

liae (Ubaldi 1995) Blasi, Di Pietro \& Filesi 2004

Aceri obtusati-Quercetum cerridis Ubaldi \& Speranza ex Ubaldi 1995

arbutetosum unedonis subass. nova

Carpinus betulus variant

Lauro nobilis-Quercenion pubescentis Ubaldi 1995

Roso sempervirentis-Quercetum pubescentis Biondi 1986

quercetosum pubescentis Allegrezza, Baldoni, Biondi, Taffetani \& Zuccarello 2002

ericetosum multiflorae Catorci \& Orsomando 1997

Crataego laevigatae-Quercion cerridis Arrigoni 1997

Crataego laevigatae-Quercenion cerridis Blasi, Di Pietro \& Filesi in Di Pietro, Azzella \& Facioni 2010

Erico arboreae-Quercetum cerridis Arrigoni in Arrigoni, Mazzanti \& Ricceri 1990 typicum lathyretosum veneti subass. nova Cephalanthero longifoliae-Quercetum cerridis Scoppola \& Filesi 1998

Castanea sativa variant

Malo florentinae-Quercetum frainetto Biondi, Gigante, Pignattelli \& Venanzoni 2001 viburnetosum tini Biondi, Gigante, Pignattelli \& Venanzoni 2001 


\section{REFERENCES}

Agnelli, A., Allegrezza, M., Biondi, E., Cocco, S., Corti, G. \& Pirchio, F. 2008: Pedogenesi e paesaggio vegetale: il ruolo dell'esposizione. Fitosociologia 45(1): 23-28.

Allegrezza, M. 2003: Vegetazione e paesaggio vegetale della dorsale del Monte San Vicino (Appennino centrale). Fitosociologia 40 (1) suppl. 1: 3-118.

Allegrezza, M., Baldoni, M., Biondi, E., Taffetani, F. \& Zuccarello, V. 2002: Studio fitosociologico dei boschi a Quercus pubescens s.l. delle Marche e di alcune zone contigue dell'Appennino centro-settentrionale (Italia centrale). Fitosociologia 39(1): 161-171.

Allegrezza, M., Biondi, E. \& Felici, S. 2006: A phytosociological analysis of the vegetation of the central Adriatic sector of the Italian peninsula. Hacquetia 5(2): 135-175.

Allegrezza, M., Biondi, E., Formica, E. \& Ballelli, S. 1997: La vegetazione dei settori rupestri calcarei dell'Italia centrale. Fitosociologia 32: 91-120.

Argenti, G., Bianchetto, E., Ferretti, F., Giulietti, V., Milandri, M., Pelleri, F., Romagnoli, P., Signorini, M. A. \& Venturi, E. 2006: Characterization of an abandoned pastoral area in the Northern Apennines, Italy. Forest@ 3(3): 387-396. URL [http://www.sisef.it/forest@/ show.php?id=385].

Arrigoni, P. V. 1997: Documenti per la carta della vegetazione delle Cerbaie (Toscana settentrionale). Parlatorea 2: 39-71.

Arrigoni, P. V. \& Bartolini, L. 1997: Documenti per la carta della vegetazione della Calvana di Prato in Toscana. Parlatorea 2: 101-123.

Arrigoni, P. V. \& Di Tommaso, P. L. 1997: La vegetazione del Monte Argentario (Toscana meridionale). Parlatorea 2: 5-38.

Arrigoni, P. V., Foggi, B., Bechi, N. \& Ricceri, C. 1997: Documenti per la carta della vegetazione di Monte Morello (Provincia di Firenze). Parlatorea 2: 73-100.

Arrigoni, P. V., Mazzanti, A. \& Ricceri, C. 1990: Contributo alla conoscenza dei boschi della Maremma grossetana. Webbia 44(1): 121-150.

Biondi, E. 1986: La vegetazione del Monte Conero (con carta della vegetazione in scala 1: 10.000). Regione Marche. Assessorato Urbanistica e Ambiente. Tecnostampa, Ostra Vetere (Ancona).

Biondi, E. \& Allegrezza, M. 1996: Il paesaggio ve- getale del territorio collinare anconetano. Giorn. Bot. Ital. 130(1): 117-135.

Biondi, E., Ballelli, S., Allegrezza, M. \& Manzi, A. 1990: La vegetazione dei calanchi di Gessopalena (Abruzzo meridionale). Doc. Phytosoc. 12: 257-263.

Biondi, E., Brugiapaglia, E., Allegrezza, M. \& Ballelli, S. 1992: La vegetazione del littorale marchigiano (Adriatico centro-settentrionale). Coll. Phytosoc. 19: 429-460.

Biondi, E., Casavecchia, S., Pinzi, M., Allegrezza, M. \& Baldoni, M. 2002a: The syntaxonomy of the mesophilous woods of the Central and Northern Apennines (Italy). Fitosociologia 39(2): 71-93.

Biondi, E., Casavecchia, S. \& Gigante, D. 2003: Contribution to the syntaxonomic knowledge of the Quercus ilex L. woods of the central European Mediterranean basin. Fitosociologia 40(1) 129-156.

Biondi, E., Feoli, F. \& Zuccarello, V. 2004: Modelling environmental responses of plant associations: a review of some critical concepts in vegetation study. Critical Reviews in Plant Sciences 23(2): 149-156.

Biondi, E., Gigante, D., Pignattelli, S. \& Venanzoni, R. 2001: I boschi a Quercus frainetto Ten. presenti nei territori centro-meridionali della penisola italiana. Fitosociologia 38(2): 97-111.

Biondi, E., Gigante, D., Pignattelli, S. \& Venanzoni, R. 2002b: I boschi del piano collinare della Provincia di Terni. Fitosociologia 39(1): 135-160.

Biondi, E., Orsomando, E., Baldoni, M. \& Catorci, A., 1995: Le cerrete termofile del Comprensorio Trasimeno. Ann. Bot. (Roma), 51. Studi sul Territorio Suppl. 10(1): 195-210.

Blasi, C. \& Di Pietro, R. 1998: Two new phytosociological types of Quercus pubescens s.l. woodland communities in southern Latium. Plant Biosystems 132(3): 207-223.

Blasi, C., Di Pietro, R. \& Filesi, L. 2004: Syntaxonomical revision of Quercetalia pubescenti-petraeae in the Italian Peninsula. Fitosociologia 41 (1): 87-164.

Braun-Blanquet, J. 1964: Pflanzensoziologie. $3^{\text {rd }}$ ed. Springer, Wien - New York. 865 pp.

Carranza, M. L., Acosta, A. T. R., Stanisci, A., Pirone, G. \& Ciaschetti, G. 2008: Ecosystem classification for EU habitat distribution assessment in sandy coastal environments: an application in central Italy. Environ. Monit. Assess. 140: 99-107. 
Casini, S., Chiarucci, A. \& De Dominicis, V. 1995: Phytosociology and ecology of the Chianti woodlands. Fitosociologia 29: 115-136.

Casini, S. \& De Dominicis, V. 1999: Memoria illustrativa per la carta della vegetazione del Chianti (scala 1: 50.000). Studio fitosociologico. Parlatorea 3: 79-106.

Catorci, A., Farabollini, P., Orsomando, E. \& Pambianchi, G. 1993: Sulla distribuzione dei paleosuoli fersiallitici e dei boschi a Quercus cerris L. nel territorio del Foglio n. 324 (Foligno). Studi per l'Ecologia del Quaternario 15: 95-99.

Catorci, A. \& Orsomando, E. 1997: Roso sempervirentis-Quercetum pubescentis Biondi 1986 nelle Colline Premartane (Umbria - Italia centrale). Fitosociologia 32: 213-220.

Catorci, A. \& Orsomando, E. 1998: Aspetti corologici e fitosociologici di Quercus frainetto Ten. in Umbria. Fitosociologia 35(1): 51-63.

Catorci, A. \& Orsomando, E. 2001: Note illustrative della Carta della Vegetazione del Foglio Nocera Umbra (N. 312 - Carta d'Italia I.G.M. - 1: 50.000). Braun-Blanquetia 23: 1-129.

Catorci, A., Orsomando, E. \& Pambianchi, G. 1994: Relation between vegetational types and rocky substratum in the Umbria and Marche regions. Boll. A.I.C., 90-91: 35-40.

Catorci, A., Vitanzi, A., Paura, B., Iocchi, M. \& Ballelli, S. 2008: La vegetazione forestale dei substrati arenacei della Val d'Aso (Marche, Italia centrale). Fitosociologia 45(2): 41-76.

Conti, F., Abbate, G., Alessandrini, A. \& Blasi, C. (eds.) 2005: An annotated checklist of the Italian vascular flora. Palombi Editori, Roma. 420 pp.

Cremaschi, M. \& Rodolfi, G. 1991: Il suolo. La Nuova Italia Scientifica. Roma. 427 pp.

Decocq M. 2000: The "masking effect" of silviculture on substrate-induced plant diversity in oak-hornbeam forests from northern France. Biodiversity and Conservation 9: 1467-1491.

Di Pietro, R., Azzella, M. M., Facioni, L. 2010: The forest vegetation of the Tolfa-Ceriti Mountains (Northern Latium-Central Italy). Hacquetia 9(1): 91-150.

Foggi, B., Chegia, B. \& Viciani, D. 2006: Contributo alla conoscenza della vegetazione del Promontorio di Piombino. Parlatorea 8: 121-139.

Foggi, B., Selvi, F., Viciani, D., Bettini, D. \& Gabelli, A. 2000: La vegetazione forestale del bacino del fiume Cecina (Toscana centro-occidentale). Parlatorea 4: 39-73.
Géhu, J. M. \& Rivas-Martínez, S. 1981: Notions fondamentales de phytosociologie. Ber. Int. simp. Int. Vereinigung Vegetationsk: 5-33.

Giovagnotti, C., Calandra, R., Leccese, A. \& Giovagnotti, E. 2003: I Paesaggi Pedologici e la Carta dei Suoli dell'Umbria. Camera di Commercio, Industria, Artigianato e Agricoltura di Perugia. $192 \mathrm{pp}$.

McCune, B. \& Grace, J.B. 2002: Analysis of Ecological Communities. MjM Software Design. Gleneden Beach, Oregon. 300 pp.

McCune, B. \& Mefford, M.J. 2006: PC-ORD. Multivariate Analysis of Ecological Data. Version 5. MjM Software Design. Gleneden Beach, Oregon.

Mitrakos, K. 1980: A theory for Mediterranean plant life. Acta Oecologica/Oecologia Plantarum 1(15), 3: 245-252.

Mitrakos, K. 1982: Winter low temperatures in mediterranean-type ecosystems. Ecologia Mediterranea 8(1-2): 95-102.

Orloci, L. 1978: Multivariate analysis in vegetation research. W. Junk, The Hague. 481 pp.

Orsomando, E. \& Catorci, A. 2000: The phytoclimate of Umbria. Parlatorea 6: 5-24.

Pieruccini, P. 2007: Suoli e geomorfologia delle praterie montane nell'Appennino Umbro-Marchigiano. In: Catorci, A. \& Gatti, R. (eds.): Le praterie montane dell'Appennino maceratese. Braun-Blanquetia 42: 19-35.

Pignatti, S. 1982: Flora d'Italia. Voll. 1-3 Edagricole, Bologna, 2302 pp.

Pignatti, S. 2005: Valori di bioindicazione delle piante vascolari della flora d'Italia. BraunBlanquetia 39: 1-97.

Pirone, G. 1992: Lineamenti vegetazionali della Maiella. Quaderni di Abruzzo. La valle dell' Orte (ambiente, cultura, società) 14: 31-50.

Podani, J. 2001: SYN-TAX 2000. Computer program for data analysis in ecology and systematics. User's manual. Scientia Publishing, Budapest. 53 pp.

Rivas-Martínez, S. 2004: Global bioclimatics (Clasificación bioclimática de la Tierra). Phytosociological Research Center, Madrid. URL [http://www.globalbioclimatics.org (version 23/04/2004)].

Rivas-Martínez, S., Díaz, T.E., Fernández-Gonzáles, F., Izco, J., Loidi, J., Lousã, M. \& Penas, A. 2002: Vascular plant communities of Spain and Portugal. Addenda to the syntaxonomical checklist of 2001. Itinera Geobotanica 15 (1-2): 5-922. 
Rivas-Martínez, S., Fernández-González, F., Loidi, J., Lousã, M. \& Penas, A. 2001: Syntaxonomical checklist of vascular plant communities of Spain and Portugal to association level. Itinera Geobotanica 14: 5-341.

Scoppola, A. 1998: La vegetazione della Riserva Naturale del Monte Rufeno (VT). Regione Lazio, Assessorato U.T.V. delle risorse ambientali. Riserva Naturale Monte Rufeno. Comune di Acquapendente. Stabilimento Tipolitografico La Commerciale, Acquapendente, $88 \mathrm{pp}$.

Scoppola, A. \& Filesi, L. 1998: Sui querceti del Lathyro montani-Quercion cerridis dell'alto Lazio. Ann. Bot. (Roma) 54(3): 295-301.

Servizio Geologico d'Italia 1980: Carta Geologica dell'Umbria (Scala 1: 250.000). Regione dell'Umbria. Giunta Regionale. Dipartimento per l'assetto del territorio. L.A.C., Firenze.

SPSS Inc. 2005: SPSS for Windows. Version 13.0.1. Chicago, Illinois, NJ, USA.

Taffetani, F. 2000: Serie di vegetazione del complesso geomorfologico del Monte dell'Ascensione (Italia centrale). Fitosociologia 37(1): 93-151.

Taffetani, F., Zitti, S. \& Giannangeli, A. 2004: Vegetazione e paesaggio vegetale della Dorsale di Cingoli (Appennino Centrale, Dorsale Marchigiana). Fitosociologia 41(2) suppl. 1: 83-161.

Tardella, F.M., Vitanzi, A., Gatti, R., Cesaretti, S. \& Catorci, A. 2010: Il paesaggio vegetale della dorsale del Monte S. Pancrazio (Umbria - Italia centrale). Fitosociologia 47(1): 65-101.
Tutin, T. G., Heywood, V. H., Burges, N. A., Moore, D. M., Valentine, D. H., Walters, S. M. \& Webb, D. A. (eds.) 1964-1980: Flora Europaea. Voll. 1-5. Cambridge University Press, 2248 pp.

Tutin, T. G., Burges, N. A., Chater, A. O., Edmonson, J. R., Heywood, V. H., Moore, D. M., Valentine, D. H., Walters, S. M. \& Webb, D. A. (eds.) 1993: Flora Europaea. Vol. 1. $2^{\text {nd }} \mathrm{ed}$. Cambridge University Press, 582 pp.

Ubaldi D. 1988: La vegetazione boschiva della provincia di Pesaro e Urbino. Esercitazioni della Accademia Agraria in Pesaro. Serie 3, Vol. 20: 99-192.

Ubaldi, D. \& Speranza, M. 1982: L'inquadramento sintassonomico dei boschi a Quercus cerris ed Ostrya carpinifolia del Flysch nell'Appennino marchigiano settentrionale. Studia Geobotanica 2: 123-140.

van der Maarel, E. 1979: Transformation of coverabundance values in phytosociology and its effects on community similarity. Vegetatio 39: 97-144.

Westhoff, V. \& van der Maarel, E. 1978: The Braun-Blanquet approch: $2^{\text {nd }}$ ed. In: Whittaker, R. H. (ed.): Classification of Plant Communities. W. Junk, The Hague, pp. 287-399.

Zuccarello, V., Allegrezza, M., Biondi, E. \& Calandra, R. 1999: Valenza ecologica di specie e di associazioni prative e modelli di distribuzione lungo gradienti sulla base della teoria degli insiemi sfocati (fuzzy set theory). BraunBlanquetia 16: 121-225.

Received 26. 5. 2010

Revision received 27. 10. 2010 Accepted 28. 10. 2010 


\section{APPENDIX}

\section{Localities, date of the relevés and accidental species}

Table 2: Erico arboreae-Quercetum cerridis Arrigoni in Arrigoni, Mazzanti \& Riccieri 1990

typicum (rel. 1-12)

lathyretosum veneti subass. nova (rel. 13-15)

Locality and date of the relevés: rel. 1-3

- Between Gualdo Cattaneo and Colle Martino (11/06/2005); rel. 4-5 - Colle Martino (04/06/ 2005); rel. 6 - Colle Martino (25/06/2006);rel. 7 - Between Colle Mariano and Colle S. Ruffino (29/06/2005); rel. 8-9 - Colle Mariano (29/ 06/2005); rel. 10 - Colle Mariano (29/06/2005); rel. 11-12 - Madonna del Monte (25/06/2006); rel. 13-15 - Between Gualdo Cattaneo and Colle Martino (18/06/2005).

Accidental species: rel. 5 - Cistus salviifolius L., +; Inula conyzae (Griess.) Meikle, +; rel. 12 Geranium dissectum L., +; rel. 13 - Cruciata laevipes Opiz, +; Geranium sanguineum L., +; Vicia tenuifolia Roth subsp. tenuifolia, +; rel. 15 - Asplenium trichomanes L. subsp. quadrivalens D.E. Mey, +.

Table 3: Cephalanthero longifoliae-Quercetum cerridis Scoppola \& Filesi 1998

Castanea sativa variant (rel. 6-11)

Locality and date of the relevés: rel. 1-4 - Madonna della Valle (11/06/2005); rel. 5 - C. S. Benedetto (08/06/2007); rel. 6 - Madonna del Monte (06/07/2006); rel. 7 - Madonna della Valle (06/06/2007); rel. 8 - Madonna del Monte $(15 / 06 / 2007)$; rel. 9 - Madonna del Monte $(06 / 06 / 2007)$; rel. 10 - Poggio della Botte (06/06/2007); rel. 11 - Hydrographic left of Rio delle Forche (15/06/2007).

Accidental species: rel. 2 - Dactylis glomerata L. subsp. glomerata,+ ; rel. 3 - Cynosurus cristatus L., +; Vicia hybrida L., +; Vicia loiseleurii Boiss., +; rel. 7 - Carex flacca Schreb. (s.l.).

Table 5: Aceri obtusati-Quercetum cerridis Ubaldi \& Speranza ex Ubaldi 1995

arbutetosum unedonis subass. nova (rel. 1-15)

Carpinus betulus variant (rel. 16-17)

Locality and date of the relevés: rel. 1-2 - Civitelle (11/06/2005); rel. 3 - C. S. Benedetto (08/06/2005); rel. 4-6 - Civitelle (18.06.2005); rel. 7 - C. S. Benedetto (06/06/2005); rel. 8-9 - Civitelle (08/06/2005); rel. 10 - Colle del Pino (08/06/2005); rel. 11 - Civitelle (18/06/2005); rel.
12-13 - Colle di Luna (29/06/2005); rel. 14 - C. Alba (29/06/2005); rel. 15 - Poggio della Botte (15/06/2006); rel. 16 - Along Sambro stream (25/ 06/2005); rel. 17 - Along Sambro stream (25/ 06/2005).

Accidental species: rel. 8 - Sedum cepaea L., +; rel. 13 - Geranium sanguineum L., +; rel. 14 - Cruciata laevipes Opiz, +; rel. 16 - Inula salicina L., +; Pteridium aquilinum (L.) Kuhn, +.

Table 6: Malo florentinae-Quercetum frainetto Biondi, Gigante, Pignattelli \& Venanzoni 2001 viburnetosum tini Biondi, Gigante, Pignattelli \& Venanzoni 2001

Locality and date of the relevés: rel. 1-3 (rel. 1-3 of Table 4 in Catorci \& Orsomando 1998) - Collestrada (09/09/1996); rel. 4-8 (rel. 7-11 of Table 2 in Biondi et al. 2001) - Collestrada (15/05/1997).

Table 7: Cyclamino hederifolii-Quercetumilicis Biondi, Casavecchia \& Gigante 2003

quercetosum cerridis subass. nova (rel. 1-9)

cyclaminetosum hederifolii Biondi, Casavecchia \& Gigante 2003 (rel. 10-19)

Locality and date of the relevés: rel. 1-2 - Pomonte (18/06/2005); rel. 3 - Between C. Fonte Trosciano and C. la Botte (24/04/2008); rel. 4 Between Colle di Luna and C. Alba (29/06/2005); rel. 5 - Poggio Valdesco (29/06/2007); rel. 6 - Monte Perugia Vecchia (29/06/2007); rel. 7 Poggio delle Civitelle (01/06/2007); rel. 8 - Sotto Colle di Luna, near the lake (29/06/2005); rel. 9 - Pomonte (18/06/2005); rel. 10 - Poggio Montone (25/06/2005); rel. 11 - Poggio Montone (24/04/2008); rel. 12 - Poggio Femmina Morta (25/06/2005); rel. 13 - Between Poggio Montone and Poggio Femmina Morta (25/06/2005); rel. 14-15 - C. Torre Burchio (25/06/2005); rel. 16 Between Poggio Montone and Poggio Femmina Morta (25/06/2005); rel. 17 - Poggio Belvedere (24/04/2008); rel. 18 - Monte Veduta del Lago (24/04/2008); rel. 19 - Monte Perugia Vecchia (09/06/2006).

Table 9: Roso sempervirentis-Quercetum pubescentis Biondi 1986

ericetosum multiflorae Catorci \& Orsomando 1997 (rel. 1-6)

quercetosum pubescentis Allegrezza, Baldoni, Biondi, Taffetani \& Zuccarello 2002 (rel. 7-10)

Locality and date of the relevés: rel. 1 (rel. 1 of Table 1 in Catorci \& Orsomando 1997) - Loc. 
Capretta (19/07/1996); rel. 2 (rel. 3 of Table 1 in Catorci \& Orsomando 1997) - Loc. Casenove di Uncinano (19/07/1996); rel. 3 (rel. 5 of Table 1 in Catorci \& Orsomando 1997) - Colle Martino (20/07/1996); rel. 4 (rel. 2 of Table 1 in Catorci \& Orsomando 1997) - Colle S. Paolo (19/07/1996); rel. 5 (rel. 7 of Table 1 in Catorci \& Orsomando 1997) - S. Gregorio (20/07/1996); rel. 6 (rel. 4 of Table 1 in Catorci \& Orsomando 1997) - Colle
S. Filippo (20/07/1996); rel. 7 (rel. 6 of Table 1 in Catorci \& Orsomando 1997) - S. Gregorio (20/07/1996); rel. 8 (rel. 9 of Table 1 in Catorci \& Orsomando 1997) - Colle Cigliane (18/06/1996); rel. 9 (rel. 8 of Table 1 in Catorci \& Orsomando 1997) - Road leading to Canalicchio (10/08/1996); rel. 10 (rel. 10 of Table 1 in Catorci \& Orsomando 1997) - Monte Caciolfo (18/06/1996).

Table 1: Main features of the study area bioclimatic belts. Termotype and Ombrotype classification follows Rivas-Martínez (2004); for number of months of aridity and cold stress calculation Mitrakos' indexes $(1980,1982)$ were applied (from: Orsomando \& Catorci 2000, modified).

Tabela 1: Glavne značilnosti bioklimatskih pasov obravnavanega območja. Termotipska in ombrotipska uvrstitev sledi Rivas-Martínezu (2004); za število sušnih mesecev in mraza smo uporabili indekse po Mitrakosu (1980, 1982) (iz: Orsomando \& Catorci 2000, spremenjeno).

\begin{tabular}{|c|c|c|c|c|c|c|c|c|c|c|}
\hline 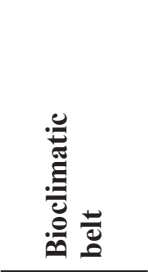 & 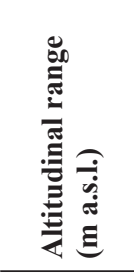 & 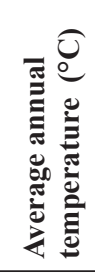 & 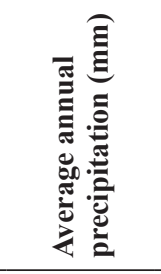 & 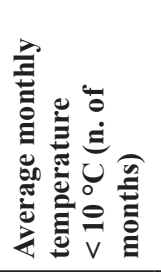 & 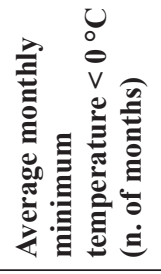 & 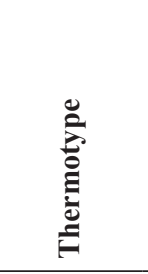 & 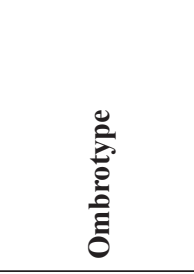 & 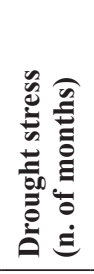 & 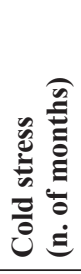 & 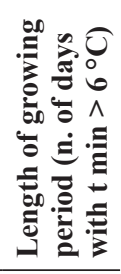 \\
\hline $\begin{array}{l}\text { Lower } \\
\text { Meso- } \\
\text { temperate }\end{array}$ & $\begin{array}{c}200 / 250- \\
550 / 600\end{array}$ & $13-14$ & $800-1000$ & $4-5$ & $0-1$ & $\begin{array}{l}\text { Lower } \\
\text { Meso- } \\
\text { temperate }\end{array}$ & $\begin{array}{c}\text { Upper } \\
\text { Subhumid }\end{array}$ & 1 & 6 & $180-210$ \\
\hline $\begin{array}{c}\text { Upper } \\
\text { Meso- } \\
\text { temperate }\end{array}$ & $\begin{array}{c}550 / 600- \\
900 / 950\end{array}$ & $11-13$ & $1000-1100$ & $5-6$ & $1-2$ & $\begin{array}{c}\text { Upper } \\
\text { Meso- } \\
\text { temperate }\end{array}$ & $\begin{array}{c}\text { Upper } \\
\text { Subhumid/ } \\
\text { Lower Humid }\end{array}$ & 0 & $7-8$ & $<180$ \\
\hline
\end{tabular}




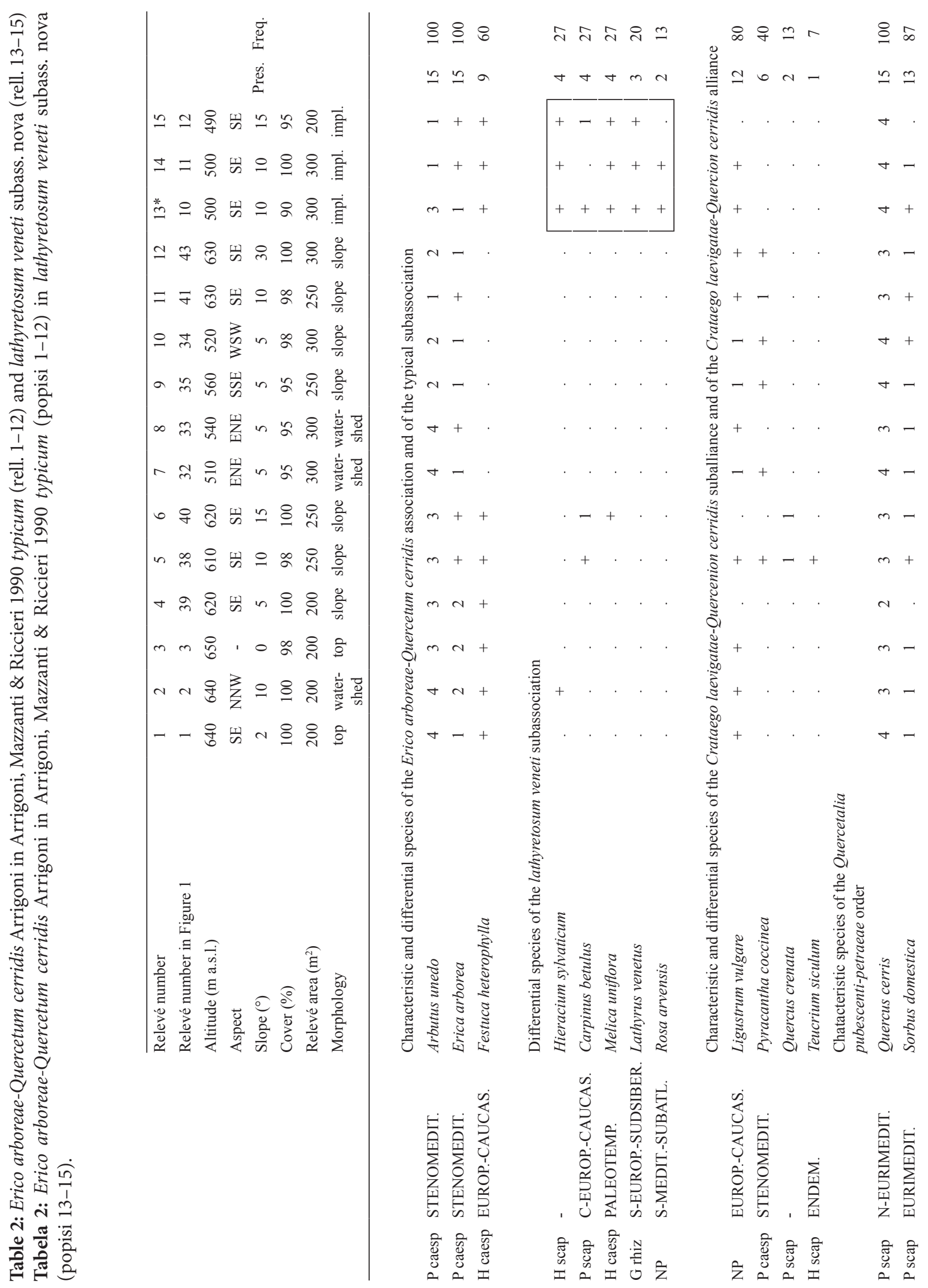




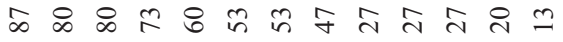

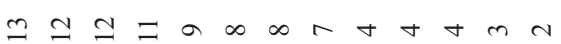
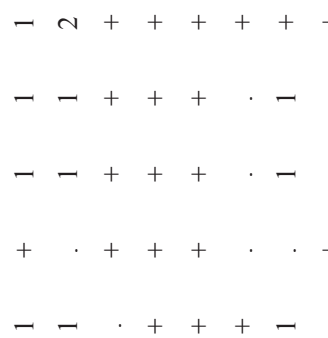

$-+++++++++$

$\sim-+++-$

N

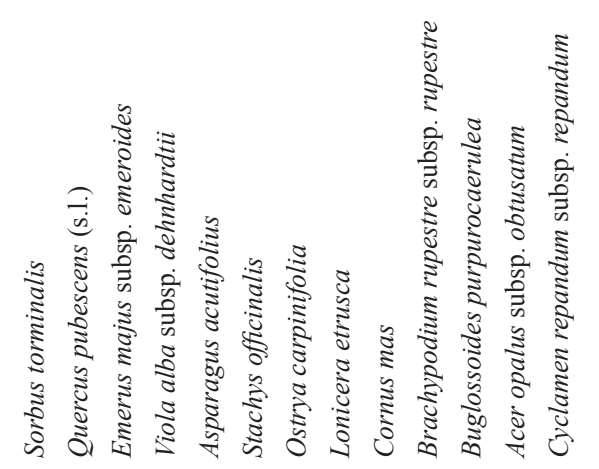

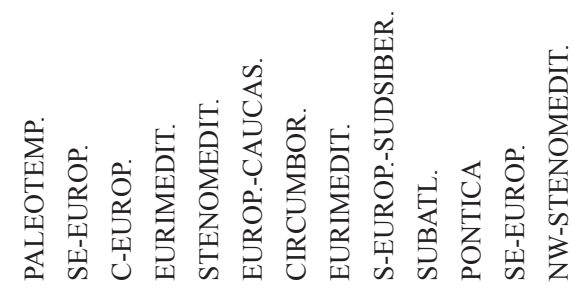

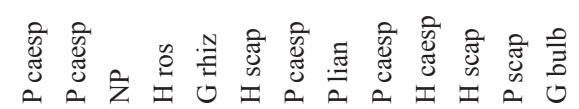

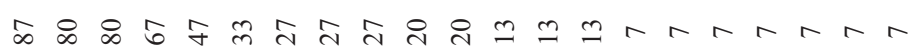

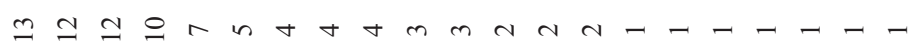

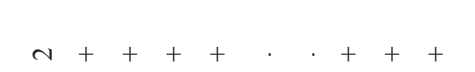

$\sim++$

ข

n. ++

N

$v+++$

$-+$

N-

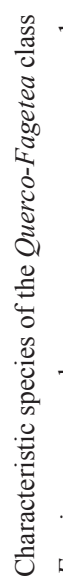
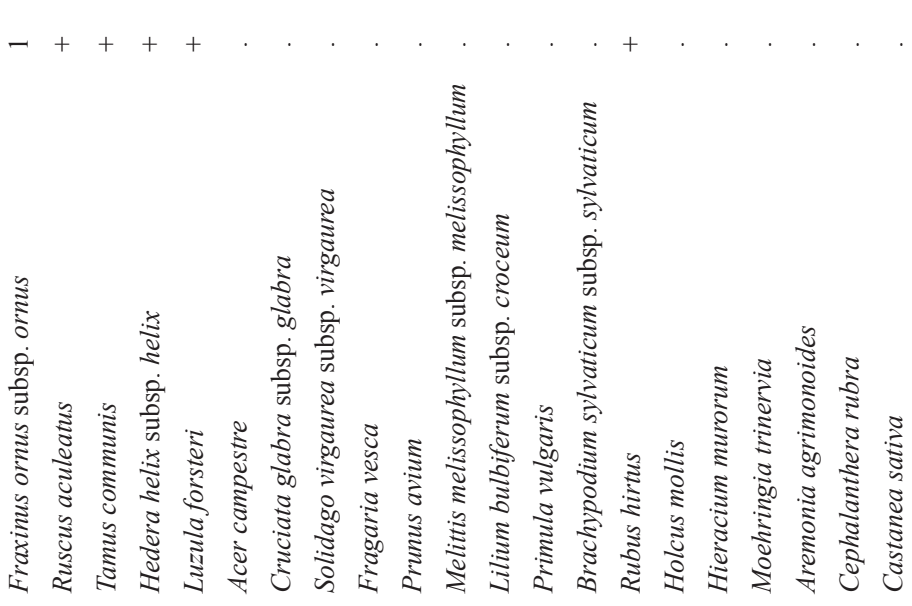

㟧

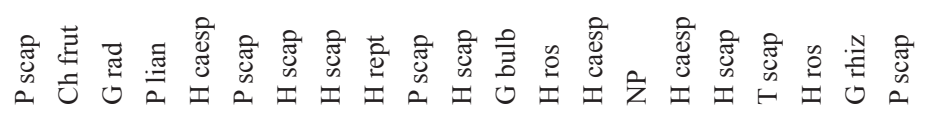




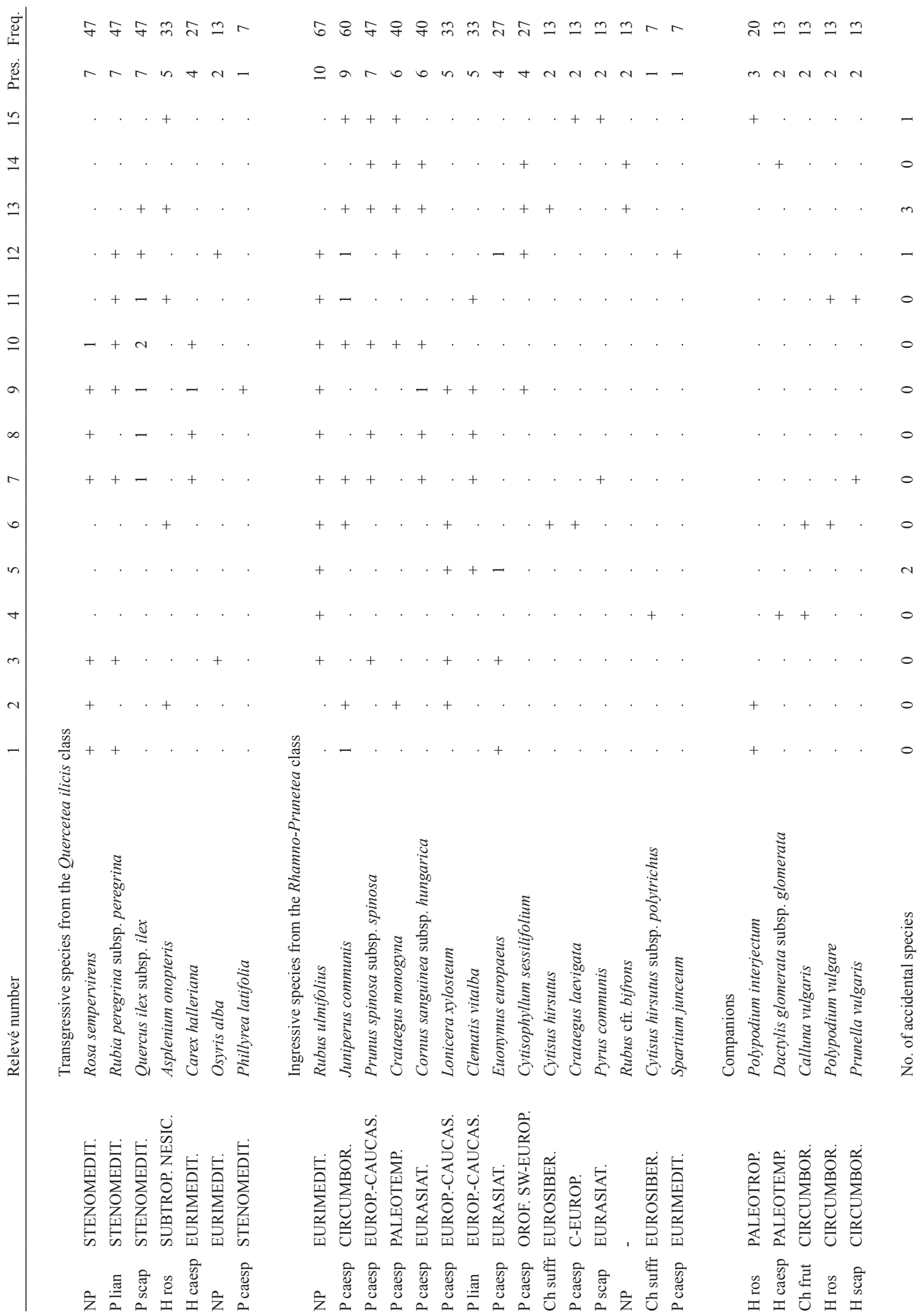


Table 3: Cephalanthero longifoliae-Quercetum cerridis Scoppola \& Filesi 1998. Castanea sativa variant (rel. 6-11). Tabela 3: Cephalanthero longifoliae-Quercetum cerridis Scoppola \& Filesi 1998. Castanea sativa variant (popisi 6-11).

\begin{tabular}{|c|c|c|c|c|c|c|c|c|c|c|c|c|}
\hline Relevé number & 1 & 2 & 3 & 4 & 5 & 6 & 7 & 8 & 9 & 10 & 11 & \\
\hline Relevé number in Figure 1 & 4 & 5 & 6 & 7 & 47 & 43 & 45 & 49 & 44 & 46 & 48 & \\
\hline Altitude (m a.s.l.) & 620 & 620 & 640 & 640 & 520 & 620 & 630 & 630 & 630 & 630 & 520 & \\
\hline Aspect & $\mathrm{NE}$ & $\mathrm{NE}$ & $\mathrm{NE}$ & $\mathrm{NE}$ & ESE & NNW & $\mathrm{NE}$ & $\mathrm{NE}$ & $\mathrm{N}$ & $\mathrm{N}$ & $\mathrm{E}$ & \\
\hline Slope $\left({ }^{\circ}\right)$ & 15 & 15 & 5 & 5 & 15 & 5 & 15 & 15 & 25 & 20 & 5 & 总总 \\
\hline Cover $(\%)$ & 95 & 100 & 100 & 100 & 100 & 100 & 100 & 100 & 98 & 100 & 100 & \\
\hline Relevé area $\left(\mathrm{m}^{2}\right)$ & 200 & 200 & 300 & 250 & 200 & 250 & 300 & 300 & 300 & 300 & 200 & \\
\hline Morphology & $\frac{\tilde{O}}{\frac{0}{n}}$ & $\frac{\tilde{a}}{\frac{o}{n}}$ & क्ष & sి & $\frac{\tilde{a}}{\frac{0}{n}}$ & 옹 & $\frac{\tilde{a}}{\frac{o}{n}}$ & $\begin{array}{l}\frac{O}{0} \\
\frac{0}{n}\end{array}$ & $\frac{\tilde{O}}{\frac{0}{n}}$ & $\frac{\tilde{a}}{\frac{0}{n}}$ & $\frac{\tilde{a}}{\frac{0}{n}}$ & \\
\hline
\end{tabular}

$\begin{array}{ll}\text { NP } & \text { S e C-EUROP. } \\ \text { H scap } & \text { EUROP.-CAUCAS. } \\ \text { NP } & \text { S-MEDIT.-SUBATL. } \\ \text { G rhiz } & \text { EURASIAT. } \\ \text { G bulb } & \text { W-STENOMEDIT. } \\ \text { H caesp } & \text { CIRCUMBOR. }\end{array}$

P scap SE-EUROP.

$\begin{array}{lll}\text { P caesp } & \text { STENOMEDIT. } & \text { Arbutus unedo } \\ \text { NP } & \text { EUROP.-CAUCAS. } & \text { Ligustrum vulgare } \\ \text { P caesp } & \text { STENOMEDIT. } & \text { Erica arborea } \\ \text { P caesp } & \text { STENOMEDIT. } & \text { Pyracantha coccinea } \\ \text { H scap } & \text { EUROP.-CAUCAS. } & \text { Rumex sanguineus } \\ \text { H scap } & \text { EUROSIBER. } & \text { Silene flos-cuculi } \\ \text { H scap } & \text { ENDEM. } & \text { Teucrium siculum } \\ \text { H ros } & \text { S-EUROP.-SUDSIBER. } & \text { Silene viridiflora }\end{array}$

Characteristic and differential species of the Cephalanthero longifoliae-Quercetum cerridis association

Rubus hirtus $\quad++++++++.+$. 873

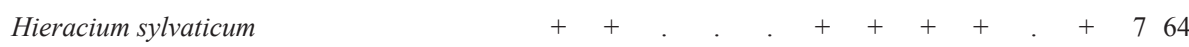

Rosa arvensis + . . . . $1+++++764$

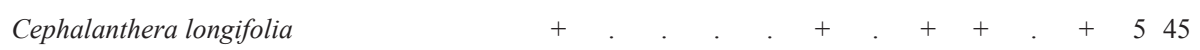

Allium pendulinum . . . . . . . . . . . . . +218

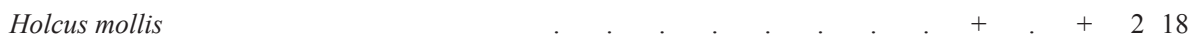

Differential species of the Castanea sativa variant

Castanea sativa

\begin{tabular}{|llll}
$21+2$ & 2 & 2 &
\end{tabular} 64

Characteristic and differential species of the Crataego laevigatae-Quercenion cerridis suballiance and of the Crataego laevigatae-Quercion cerridis alliance

Chatacteristic species of the Quercetalia pubescenti-petraeae order

$\begin{array}{lll}\text { P scap } & \text { N-EURIMEDIT. } & \text { Quercus cerris } \\ \text { P caesp } & \text { S-EUROP.-SUDSIBER. } & \text { Cornus mas } \\ \text { P scap } & \text { EURIMEDIT. } & \text { Sorbus domestica } \\ \text { P caesp } & \text { PALEOTEMP. } & \text { Sorbus torminalis } \\ \text { NP } & \text { C-EUROP. } & \text { Emerus majus subsp. emeroides } \\ \text { P caesp } & \text { CIRCUMBOR. } & \text { Ostrya carpinifolia } \\ \text { P scap } & \text { SE-EUROP. } & \text { Acer opalus subsp. obtusatum } \\ \text { G bulb } & \text { NW-STENOMEDIT. } & \text { Cyclamen repandum subsp. repandum } \\ \text { H ros } & \text { EURIMEDIT. } & \text { Viola alba } \text { subsp. dehnhardtii } \\ \text { P lian } & \text { EURIMEDIT. } & \text { Lonicera etrusca } \\ \text { P scap } & \text { EUROP. } & \text { Quercus petraea } \\ \text { H scap } & \text { EUROSIBER. } & \text { Serratula tinctoria } \text { subsp. tinctoria } \\ \text { H scap } & \text { EUROP.-CAUCAS. } & \text { Stachys officinalis } \\ \text { G rhiz } & \text { STENOMEDIT. } & \text { Asparagus acutifolius } \\ \text { G rhiz } & \text { STENOMEDIT. } & \text { Arum italicum }\end{array}$

\begin{tabular}{|c|c|c|c|c|c|c|c|c|c|c|c|}
\hline & 4 & 5 & 4 & 4 & 3 & 3 & 3 & 3 & 4 & 3 & 11100 \\
\hline & + & 1 & 1 & + & 1 & + & 1 & 1 & 1 & 1 & 11100 \\
\hline & 1 & + & 1 & + & + & 1 & + & . & + & + & 1091 \\
\hline & . & . & + & 1 & + & + & & + & . & 1 & 764 \\
\hline & + & . & . & + & . & + & . & + & + & + & 764 \\
\hline & . & 1 & . & 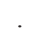 & 1 & + & 1 & . & . & 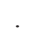 & $\begin{array}{ll}5 & 45\end{array}$ \\
\hline & . & . & 1 & . & 1 & 1 & 1 & + & . & . & $\begin{array}{ll}5 & 45\end{array}$ \\
\hline & . & . & . & . & + & + & & + & . & + & $\begin{array}{ll}5 & 45\end{array}$ \\
\hline & + & . & . & . & . & . & & + & . & + & 436 \\
\hline 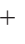 & + & + & + & . & . & . & & . & $\cdot$ & . & 436 \\
\hline & . & $\cdot$ & . & . & + & + & . & + & . & 1 & 436 \\
\hline & . & $\cdot$ & . & . & + & + & . & + & . & + & 436 \\
\hline & + & + & . & . & . & · & . & · & - & . & 327 \\
\hline & + & $\cdot$ & . & ${ }^{\prime}$ & . & . & . & . & . & & 218 \\
\hline & . & . & + & & . & . & & & & & 19 \\
\hline
\end{tabular}


Relevé number

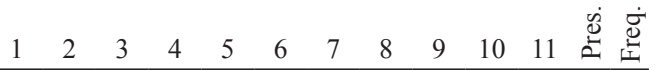

Transgressive species from the Fagetalia sylvaticae order

\begin{tabular}{|c|c|c|c|c|c|c|c|c|c|c|c|c|c|c|}
\hline P scap & C-EUROP.-CAUCAS. & Carpinus betulus & 2 & 4 & 3 & . & 1 & 4 & 4 & 3 & 4 & 4 & 1 & 10 \\
\hline H scap & EUROP.-CAUCAS. & Ranunculus lanuginosus & + & + & + & + & + & . & + & . & 1 & + & + & 98 \\
\hline P caesp & C-EUROP. & Crataegus laevigata & . & 1 & . & 1 & 1 & 1 & 1 & 1 & 1 & + & + & 98 \\
\hline H caesp & PALEOTEMP. & Melica uniflora & + & + & + & + & + & . & . & + & . & . & . & 6 \\
\hline G rhiz & SE-EUROP. & Symphytum bulbosum & . & . & . & . & . & + & + & . & + & + & . & 4 \\
\hline H scap & EURIMEDIT.-SUBATL. & Oenanthe pimpinelloides & . & . & + & . & . & + & . & + & . & . & . & 3 \\
\hline T scap & EURASIAT. & Moehringia trinervia & . & . & . & . & . & . & . & . & + & . & + & 2 \\
\hline H scap & PALEOTEMP. & Sanicula europaea & . & . & . & . & . & + & . & . & . & . & . & 1 \\
\hline Ch suffr & EUROP.-CAUCAS. & Euphorbia amygdaloides subsp. amygdaloides & . & . & . & . & . & . & + & . & . & . & . & 1 \\
\hline G par & CIRCUMBOR. & Monotropa hypopytis & . & . & . & . & . & . & . & + & . & . & . & 1 \\
\hline Ch rept & EUROP.-CAUCAS. & Vinca minor & . & . & . & . & . & . & . & . & 2 & . & . & 1 \\
\hline
\end{tabular}

P scap EUROP.-CAUCAS. Acer pseudoplatanus

Characteristic species of the Querco-Fagetea class

\begin{tabular}{|c|c|c|c|c|c|c|c|c|c|c|c|c|c|c|}
\hline Ch frut & EURIMEDIT. & Ruscus aculeatus & 2 & 3 & 3 & 3 & 1 & 3 & 3 & 1 & 2 & 1 & 2 & 11100 \\
\hline G rad & EURIMEDIT. & Tamus communis & + & + & + & + & + & + & + & + & + & + & + & 11100 \\
\hline P scap & EUROP.-CAUCAS. & Acer campestre & + & + & + & + & + & 1 & 1 & 1 & + & . & 1 & 1091 \\
\hline P lian & EURIMEDIT. & Hedera helix subsp. helix & + & 1 & + & 1 & 2 & . & 1 & 1 & 1 & + & 1 & 1091 \\
\hline H caesp & EUROP.-CAUCAS. & Festuca heterophylla & 1 & 1 & + & + & . & + & + & + & + & + & + & 1091 \\
\hline H rept & EUROSIBER. & Fragaria vesca & + & + & + & . & + & + & + & + & + & + & + & 1091 \\
\hline G rhiz & S-EUROP.-SUDSIBER. & Lathyrus venetus & + & + & + & + & ${ }^{\circ}$ & + & + & + & + & + & - & 982 \\
\hline $\mathrm{H}$ caesp & EURIMEDIT. & Luzula forsteri & + & + & + & + & . & + & . & + & + & + & + & 982 \\
\hline $\mathrm{H}$ ros & EUROP.-CAUCAS. & Primula vulgaris & + & + & . & + & + & + & + & 1 & + & + & . & 982 \\
\hline H scap & EUROSIBER. & Viola reichenbachiana & . & + & . & + & + & + & + & + & + & + & + & 982 \\
\hline$P$ caesp & SUBATL. & Daphne laureola & + & + & . & + & + & + & + & + & . & . & + & 873 \\
\hline P scap & S-EUROP.-SUDSIBER. & Fraxinus ornus subsp. ornus & 2 & . & . & 1 & . & + & + & + & 1 & . & 2 & 764 \\
\hline H rept & EUROP.-CAUCAS. & Ajuga reptans & + & + & + & . & . & + & . & . & + & + & + & 764 \\
\hline H caesp & PALEOTEMP. & Brachypodium sylvaticum subsp. sylvaticum & . & . & . & . & . & + & + & + & + & + & + & 655 \\
\hline P lian & S-EUROP.-SUDSIBER. & Lonicera caprifolium & . & . & . & . & . & + & + & + & + & + & + & 655 \\
\hline H scap & EUROSIBER. & Hieracium murorum & . & . & . & . & . & + & + & + & + & . & + & 545 \\
\hline H scap & EURASIAT. & Cruciata glabra subsp. glabra & + & + & . & . & . & . & + & . & . & + & . & 436 \\
\hline H scap & C-EUROP. & Melittis melissophyllum subsp. melissophyllum & . & . & . & . & . & + & + & . & . & + & . & 327 \\
\hline P scap & C-EUROP. & Malus sylvestris & . & + & . & + & . & . & + & . & . & . & . & 327 \\
\hline G rhiz & SE-EUROP. & Symphytum tuberosum subsp. angustifolium & + & . & + & + & . & . & . & . & . & . & . & 327 \\
\hline P caesp & EUROP.-CAUCAS. & Corylus avellana & . & . & . & . & - & . & . & . & 1 & 1 & . & 218 \\
\hline H scap & CIRCUMBOR. & Geum urbanum & . & . & . & + & . & . & . & . & . & . & . & 19 \\
\hline G bulb & OROF. C-EUROP. & Lilium bulbiferum subsp. croceum & . & . & . & . & . & + & . & . & . & . & . & 19 \\
\hline
\end{tabular}

$\begin{array}{llllllllllllllll}\text { P scap } & \text { STENOMEDIT. } & \text { Quercus ilex subsp. ilex } & + & + & 1 & + & + & + & 1 & . & 1 & + & . & 9 \\ \text { H ros } & \text { SUBTROP. NESICOLA } & \text { Asplenium onopteris } & + & + & . & . & . & . & + & . & + & + & + & 6 & 55 \\ \text { NP } & \text { STENOMEDIT. } & \text { Rosa sempervirens } & . & . & + & . & + & . & . & . & . & . & . & 2 & 18 \\ \text { P lian } & \text { STENOMEDIT. } & \text { Rubia peregrina subsp. peregrina } & . & . & . & . & . & . & . & . & . & . & + & 1 & 9\end{array}$

P caesp PALEOTEMP.

P caesp CIRCUMBOR.

P caesp EUROP.-CAUCAS.

P caesp EUROP.-CAUCAS.
Ingressive species from the Rhamno-Prunetea class

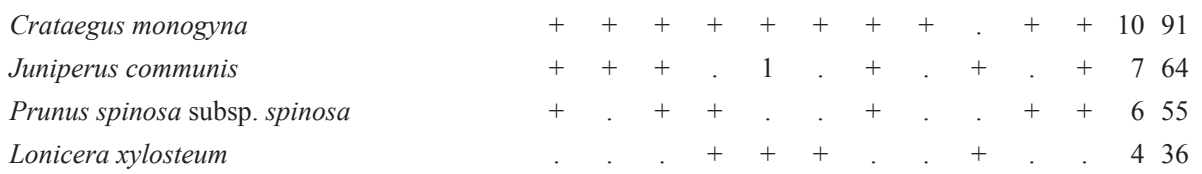




\begin{tabular}{|c|c|c|c|c|c|c|c|c|c|c|c|c|c|c|c|}
\hline & & Relevé number & 1 & 2 & 3 & 4 & 5 & 6 & 7 & 8 & 9 & 10 & 11 & & 它 \\
\hline P lian & EUROP.-CAUCAS. & Clematis vitalba & . & . & . & . & + & + & + & . & . & . & . & 32 & 27 \\
\hline$P$ caesp & EURASIAT. & Cornus sanguinea subsp. hungarica & . & + & . & . & . & + & . & . & . & . & . & 21 & 18 \\
\hline$P$ caesp & EURASIAT. & Euonymus europaeus & . & . & . & . & . & + & . & . & . & . & . & 1 & 9 \\
\hline NP & EURIMEDIT. & Rubus ulmifolius & . & . & + & . & . & . & . & . & . & . & . & 1 & 9 \\
\hline \multirow[t]{2}{*}{ P caesp } & OROF. SW-EUROP. & Cytisophyllum sessilifolium & . & . & . & . & + & . & . & . & . & . & . & 1 & 9 \\
\hline & & Companions & & & & & & & & & & & & & \\
\hline $\mathrm{H}$ ros & CIRCUMBOR. & Polypodium vulgare & . & . & . & . & + & + & + & + & + & + & + & 76 & 64 \\
\hline T scap & EURIMEDIT. ATL. & Sedum cepaea & + & + & + & + & . & . & . & . & + & . & . & 54 & 45 \\
\hline H caesp & EURIMEDIT. & Poa sylvicola & . & . & . & . & . & + & + & . & + & + & + & 54 & 45 \\
\hline H scap & EUROP.-CAUCAS. & Calamintha nepeta subsp. sylvatica & + & + & . & + & . & . & . & . & . & . & . & 32 & 27 \\
\hline H scap & CIRCUMBOR. & Prunella vulgaris & + & . & + & + & . & . & . & . & . & . & . & 32 & 27 \\
\hline H scap & OROF. S-EUROP. & Calamintha nepeta subsp. nepeta & + & + & . & + & . & . & . & . & . & . & . & 32 & 27 \\
\hline H scap & S-EUROP.-SUDSIBER. & Anthriscus nemorosa & . & + & + & + & . & . & . & . & . & . & . & 32 & 27 \\
\hline G rhiz & COSMOPOL. & Pteridium aquilinum & . & . & . & . & + & + & . & . & + & . & . & 32 & 27 \\
\hline $\mathrm{H}$ ros & PALEOTROP. & Polypodium interjectum & + & + & . & . & . & . & . & . & . & . & . & 21 & 18 \\
\hline H scap & EURASIAT. & Cruciata laevipes & . & . & . & . & . & . & . & + & . & . & + & 21 & 18 \\
\hline T scap & PALEOTEMP. & Lapsana communis & . & + & + & . & . & . & . & . & . & . & . & 21 & 18 \\
\hline $\mathrm{H}$ ros & EURIMEDIT. & Potentilla micrantha & . & . & . & . & . & + & . & . & + & . & . & 21 & 18 \\
\hline \multirow[t]{2}{*}{ Ch suffr } & C-EUROP. & Genista germanica & . & . & . & . & + & . & . & . & . & + & . & 2 & 18 \\
\hline & & No. of accidental species & 0 & 1 & 3 & 0 & 0 & 0 & 1 & 0 & 0 & 0 & 0 & & \\
\hline
\end{tabular}

Table 4: Synoptical scheme of turkey oak woods of Central Italy, referred to Melico uniflorae-Quercetum cerridis and Cephalanthero longifoliae-Quercetum cerridis, compared with those of the study area.

Column 1 - Melico uniflorae-Quercetum cerridis Arrigoni 1990 - Table 3 in Arrigoni et al. 1990; column 2 - Melico unifloraeQuercetum cerridis Arrigoni 1990 - Table 3 in Di Pietro et al. 2010; column 3 - Melico uniflorae-Quercetum cerridis Arrigoni 1990 carpinetosum betuli Arrigoni 1990 - Table 5 in Di Pietro et al. 2010; column 4 - Cephalanthero longifoliae-Quercetum cerridis Scoppola \& Filesi 1998 - Table 1 in Scoppola \& Filesi 1998; column 5 - Cephalanthero longifoliae-Quercetum cerridis Scoppola \& Filesi 1998 - Table 11 in Biondi et al. 2002; column 6 - Cephalanthero longifoliae-Quercetum cerridis Scoppola \& Filesi 1998 - Table 3, present study.

Tabela 4: Sinoptična shema cerovih gozdov srednje Italije, asociacij Melico uniflorae-Quercetum cerridis in Cephalanthero longifoliae-Quercetum cerridis, primerjani s tistimi v obravnavanem območju.

Stolpec 1 - Melico uniflorae-Quercetum cerridis Arrigoni 1990 - Tabela 3 v Arrigoni et al. 1990; stolpec 2 - Melico unifloraeQuercetum cerridis Arrigoni 1990 - Tabela 3 v Di Pietro et al. 2010; stolpec 3 - Melico uniflorae-Quercetum cerridis Arrigoni 1990 carpinetosum betuli Arrigoni 1990 - Tabela 5 v Di Pietro et al. 2010; stolpec 4 - Cephalanthero longifoliae-Quercetum cerridis Scoppola \& Filesi 1998 - Tabela 1 v Scoppola \& Filesi 1998; stolpec 5 - Cephalanthero longifoliae-Quercetum cerridis Scoppola \& Filesi 1998 - Tabela 11 v Biondi et al. 2002; stolpec 6 - Cephalanthero longifoliae-Quercetum cerridis Scoppola \& Filesi 1998 - Tabela 3, ta članek.

\begin{tabular}{lcccccc} 
Column nr. & 1 & 2 & 3 & 4 & 5 & 6 \\
Nr. of relevés & 14 & 25 & 20 & 8 & 18 & 11 \\
\hline
\end{tabular}

Characteristic and differential species of the Cephalanthero longifoliae-Quercetum cerridis association

$\begin{array}{lcccccc}\text { Rubus hirtus } & \text {. } & \text { I } & \text { I } & \text { V } & \text { I } & \text { IV } \\ \text { Allium pendulinum } & . & \text { II } & \text { II } & \text { IV } & \text {. } & \text { I } \\ \text { Rosa arvensis } & . & \text { I } & \text {. } & \text { V } & \text { V } & \text { IV } \\ \text { Cephalanthera longifolia } & . & . & . & \text { IV } & \text { III } & \text { III } \\ \text { Holcus mollis } & . & . & . & \text { III } & . & \text { I } \\ \text { Hieracium sylvaticum } & . & . & . & . & \text { II } & \text { IV }\end{array}$

\begin{tabular}{lcccccc} 
Column nr. & 1 & 2 & 3 & 4 & 5 & 6 \\
Nr. of relevés & 14 & 25 & 20 & 8 & 18 & 11 \\
\hline
\end{tabular}

Characteristic and differential species of the Melico unifloraeQuercetum cerridis association

\begin{tabular}{|c|c|c|c|c|c|}
\hline Melica uniflora & $\mathrm{V}$ & $\mathrm{V}$ & $\mathrm{V}$ & IV & II \\
\hline $\begin{array}{l}\text { Brachypodium sylvaticum subsp. } \\
\text { sylvaticum }\end{array}$ & IV & IV & III & II & IV \\
\hline Cornus mas & IV & IV & $\mathrm{V}$ & V & IV \\
\hline Oenanthe pimpinelloides & IV & IV & III & III & III \\
\hline Festuca heterophylla & IV & III & III & V & IV \\
\hline
\end{tabular}




\begin{tabular}{|c|c|c|c|c|c|c|}
\hline Column nr. & 1 & 2 & 3 & 4 & 5 & 6 \\
\hline Nr. of relevés & 14 & 25 & 20 & 8 & 18 & 11 \\
\hline Crataegus laevigata & IV & III & III & II & III & V \\
\hline $\begin{array}{l}\text { Euphorbia amygdaloides subsp. } \\
\text { amygdaloides }\end{array}$ & III & IV & IV & I & . & I \\
\hline \multicolumn{7}{|c|}{$\begin{array}{l}\text { Characteristic and differential species of the Crataego laevigatae- } \\
\text { Quercenion cerridis suballiance and of the Crataego laevigatae- } \\
\text { Quercion cerridis alliance }\end{array}$} \\
\hline Erica arborea & II & II & I & II & III & III \\
\hline Stachys officinalis & II & IV & II & $\mathrm{V}$ & IV & II \\
\hline Quercus petraea & III & II & I & $\mathrm{V}$ & II & II \\
\hline Ligustrum vulgare & I & I & II & II & . & $\mathrm{V}$ \\
\hline Malus sylvestris & I & IV & III & II & . & II \\
\hline Ranunculus lanuginosus & . & II & II & III & I & $\mathrm{V}$ \\
\hline Silene viridiflora & . & I & I & II & II & I \\
\hline Lathyrus niger & I & I & . & III & II & \\
\hline Echinops siculus & . & II & II & I & I & \\
\hline Mespilus germanica & . & II & II & I & I & \\
\hline Teucrium siculum & . & II & I & . & III & I \\
\hline Crepis leontodontoides & II & I & . & . & II & \\
\hline Pyrus communis & III & . & . & IV & II & \\
\hline Digitalis lutea subsp. australis & II & . & . & II & III & \\
\hline Cytisus scoparius & I & . & . & IV & $\mathrm{V}$ & \\
\hline Poa sylvicola & . & IV & III & . & . & III \\
\hline Cytisus villosus & . & I & III & . & II & \\
\hline Vicia grandiflora & . & I & I & . & I & \\
\hline Rumex sanguineus & . & I & II & . & . & I \\
\hline Silene flos-cuculi & . & . & I & II & . & I \\
\hline Pyracantha coccinea & I & . & . & . & . & III \\
\hline Iris foetidissima & . & II & III & . & . & \\
\hline Anthoxanthum odoratum & . & I & . & II & . & \\
\hline Arbutus unedo & . & I & . & . & . & 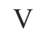 \\
\hline Poa nemoralis & . & . & . & I & I & \\
\hline
\end{tabular}

Chatacteristic species of the Quercetalia pubescenti-petraeae order Quercus cerris

Sorbus domestica

Sorbus torminalis

Viola alba subsp. dehnhardtii

Quercus pubescens (s.l.)

Lonicera etrusca

Acer opalus subsp. obtusatum

Serratula tinctoria subsp. tinctoria

Buglossoides purpurocaerulea

Ostrya carpinifolia

Scutellaria columnae

Cyclamen repandum subsp. repandum

Ilex aquifolium

Arum italicum

Emerus majus subsp. emeroides

Helleborus foetidus

IV $\mathrm{V}$ IV IV $\mathrm{V}$ II

I II II II I .

III $\mathrm{V} \quad \mathrm{V}$. IV II

$\begin{array}{lllllll}\text { I } & \text { I } & \text { I } & \text { I } & \text {. } & \text { III }\end{array}$

I I . II II II

II III II . I .

I II IV . . III

\begin{tabular}{lcccccc} 
Column nr. & 1 & 2 & 3 & 4 & 5 & 6 \\
Nr. of relevés & 14 & 25 & 20 & 8 & 18 & 11 \\
\hline Helleborus bocconei & I &. &. & & I &. \\
Hieracium racemosum &. & I &. &. & II &. \\
Allium triquetrum &. & III & I &. &. &. \\
Melica arrecta &. & I & I &. &. &. \\
Polystichum setiferum &. & I & I &. &. &. \\
Hypericum montanum &. &. &. & III & I &.
\end{tabular}

Transgressive species from the Fagetalia sylvaticae order

\begin{tabular}{|c|c|c|c|c|c|}
\hline Carpinus betulus & IV & II & $\mathrm{V}$ & III & II \\
\hline Prunus avium & II & . & . & II & I \\
\hline Carex sylvatica & II & I & II & II & . \\
\hline Campanula trachelium & II & . & I & I & . \\
\hline Anemone apennina & . & III & III & II & . \\
\hline Moehringia trinervia & . & . & I & II & . \\
\hline Loncomelos pyrenaicus & . & III & I & . & . \\
\hline Galanthus nivalis & . & I & I & . & . \\
\hline Symphytum bulbosum & . & I & . & . & . \\
\hline Sanicula europaea & . & . & I & . & . \\
\hline
\end{tabular}

Characteristic species of the Querco-Fagetea class

Hedera helix subsp. helix

$\begin{array}{llllll}\mathrm{V} & \mathrm{V} & \mathrm{V} & \mathrm{III} & \mathrm{V} & \mathrm{V}\end{array}$

Acer campestre

Cruciata glabra subsp. glabra

$\begin{array}{llllll}\mathrm{V} & \text { IV } & \mathrm{V} & \text { II } & \text { III } & \mathrm{V}\end{array}$

Fraxinus ornus subsp. ornus

IV IV I $\mathrm{V} \quad \mathrm{V}$ II

Ruscus aculeatus

Tamus communis

Viola reichenbachiana

Luzula forsteri

Daphne laureola

Melittis melissophyllum subsp.

melissophyllum

$\begin{array}{llllll}\text { IV } & \mathrm{V} & \mathrm{V} & \text { II } & \text { III } & \text { IV }\end{array}$

IV $\mathrm{V}$ V IV IV V

IV IV V IV III V

IV I II $\mathrm{V}$ II $\mathrm{V}$

III III III $\mathrm{V}$ IV V

II II III I II IV

Lathyrus venetus

$\begin{array}{llllll}\text { I } & \text { II } & \text { I } & \text { II } & \text { I } & \text { II }\end{array}$

Ajuga reptans

Fragaria vesca

Symphytum tuberosum subsp.

angustifolium

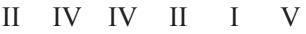

III IV IV . I IV

IV . I IV IV V

II . II IV I II

Acer monspessulanum

Primula vulgaris

Geum urbanum

Bromus ramosus

Cyclamen hederifolium

Castanea sativa

Lonicera caprifolium

II IV II . I .

Dactylorhiza maculata subsp. fuchsii

Ulmus minor subsp. minor

Platanthera clorantha

Viola riviniana

Corylus avellana

Hieracium murorum

Neottia nidus-avis
II . IV II V

. I II . II I

. I I . I .

. I II IV .

. II . II . IV

. . $\quad \mathrm{V}$ II III

. I II

. I I

. I I 


\begin{tabular}{lcccccc} 
Column nr. & 1 & 2 & 3 & 4 & 5 & 6 \\
Nr. of relevés & 14 & 25 & 20 & 8 & 18 & 11 \\
\hline
\end{tabular}

Transgressive species from the Quercetea ilicis class

Asplenium onopteris

II II IV . . III

Rosa sempervirens

II III II . . I

Rubia peregrina subsp. peregrina

II $\mathrm{V} \quad \mathrm{V}$. . I

Asparagus acutifolius

. I II . I I

Quercus ilex subsp. ilex

. I III . . V

Smilax aspera

. II III . . .

Carex olbiensis

Phillyrea latifolia

Carex distachya

. II I . .

Carex distachya

Ingressive species from the Rhamno-Prunetea class

Prunus spinosa subsp. spinosa

$\begin{array}{cccccc}\text { V } & \text { III } & \text { III } & \text { III } & \text { V } & \text { III } \\ \text { IV } & \text { IV } & \text { III } & \text { IV } & \text { IV } & \text { V } \\ \text { IV } & \text { IV } & \text { IV } & \text { II } & \text { III } & \text { I } \\ \text { II } & \text { IV } & \text { V } & . & \text { IV } & \text { I } \\ \text { IV } & \text { I } & \text { III } & . & \text { III } & \text { II } \\ \text { III } & \text { I } & . & \text { V } & \text { IV } & \text { IV } \\ . & . & \text { II } & . & \text { I } & \text { I } \\ \text { II } & . & . & . & \text { III } & . \\ \text { I } & . & . & . & . & \text { II } \\ . & . & . & . & \text { II } & \text { IV }\end{array}$

Companions

Dactylis glomerata

IV IV III IV III .

Carex flacca

$\begin{array}{lllll}\text { II } & \text { I } & \text { I } & \text { III } & \text { IV }\end{array}$

Clinopodium vulgare

II I I II III .

Pteridium aquilinum

$\begin{array}{llllll}\text { I } & \text { I } & \text { I } & \text {. } & \text { II } & \text { II }\end{array}$

Teucrium chamaedrys

I I . IV II .

Sedum cepaea

. II II II . III

Geranium sanguineum

II I . . I .

Stellaria media

. II II II . .

Potentilla micrantha

Silene italica

. II . IV . I

Prunella vulgaris

. I . III II .

Genista germanica

. . I . I II

Lapsana communis

. . . II II I

. I . . . I I




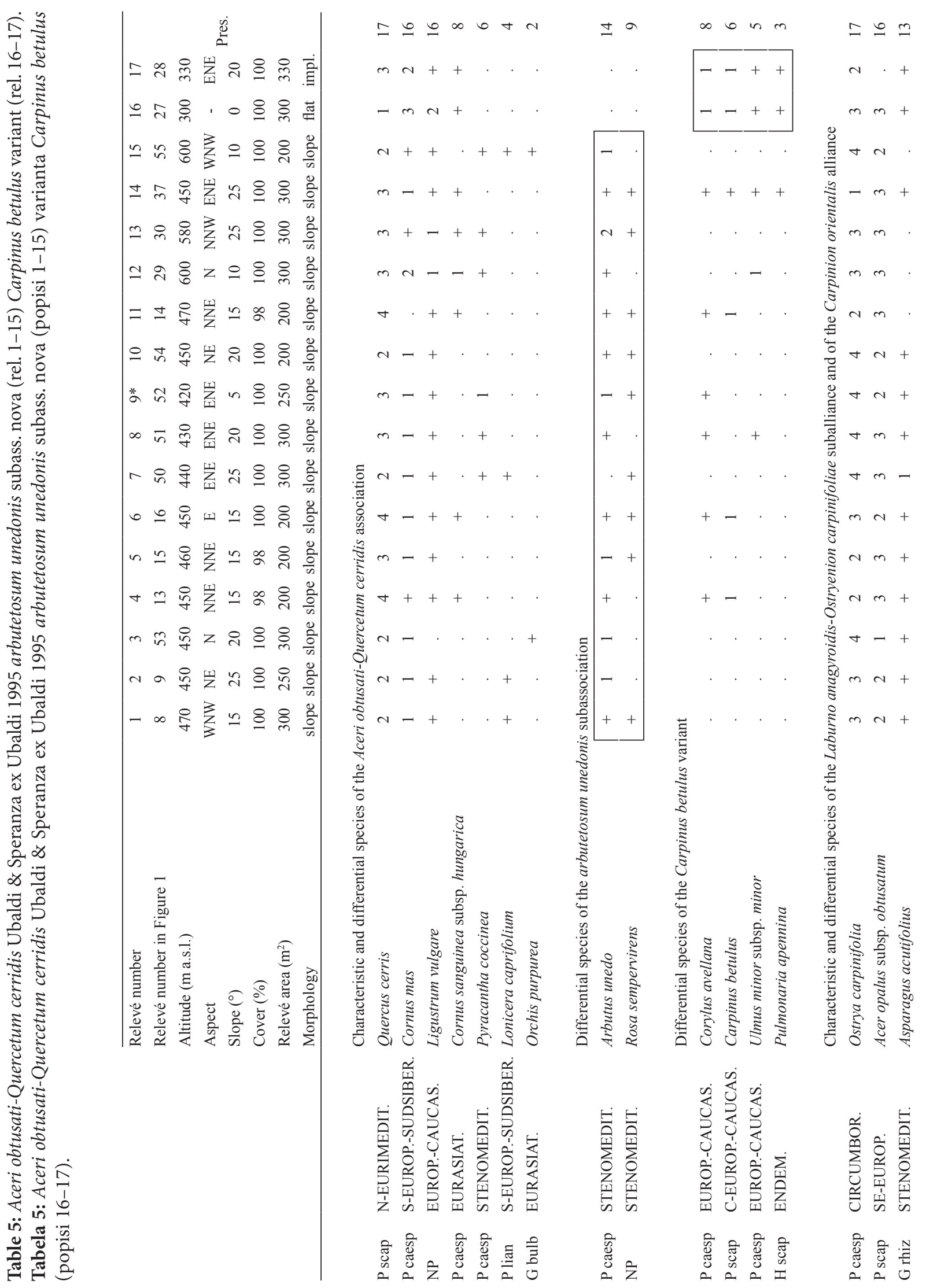




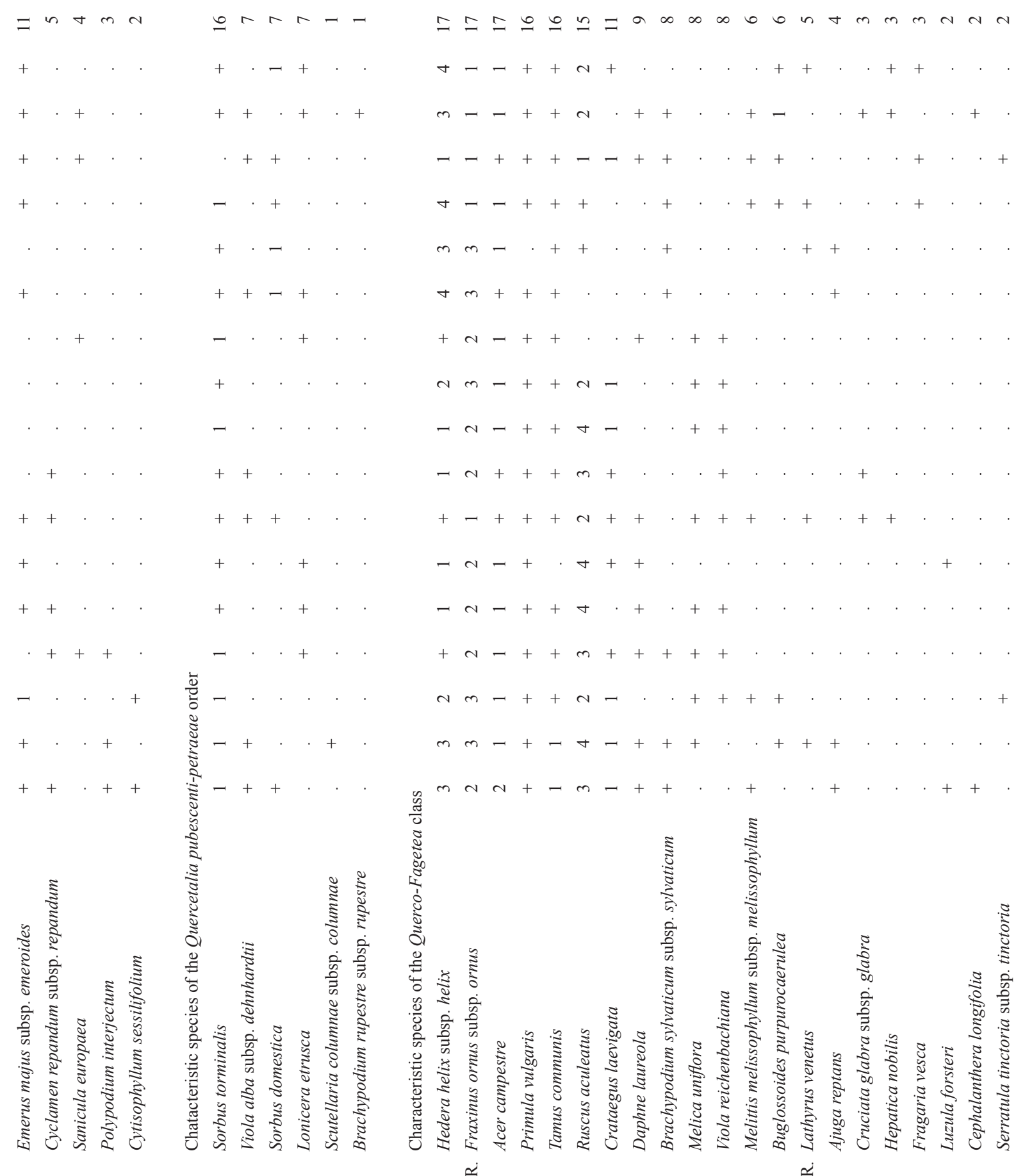

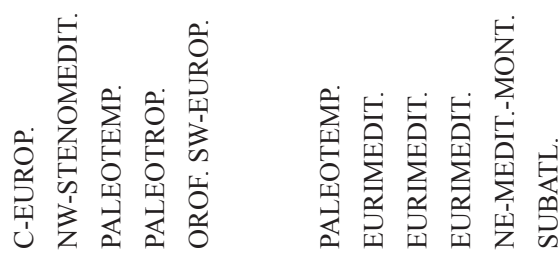

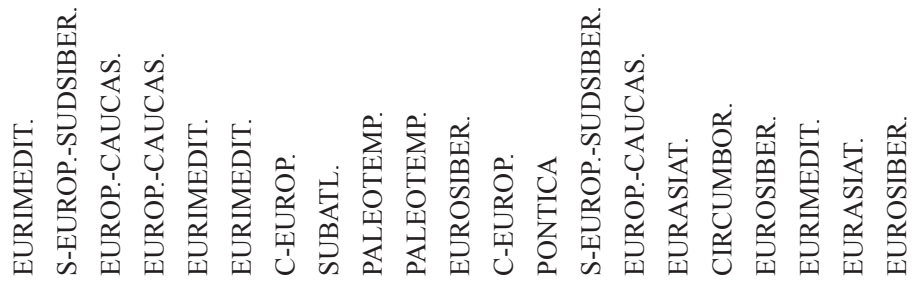

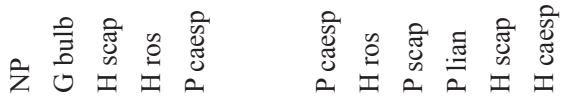

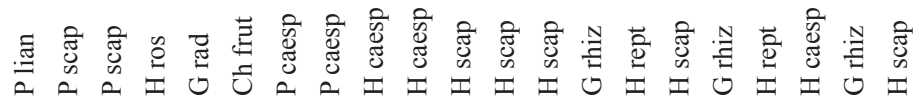



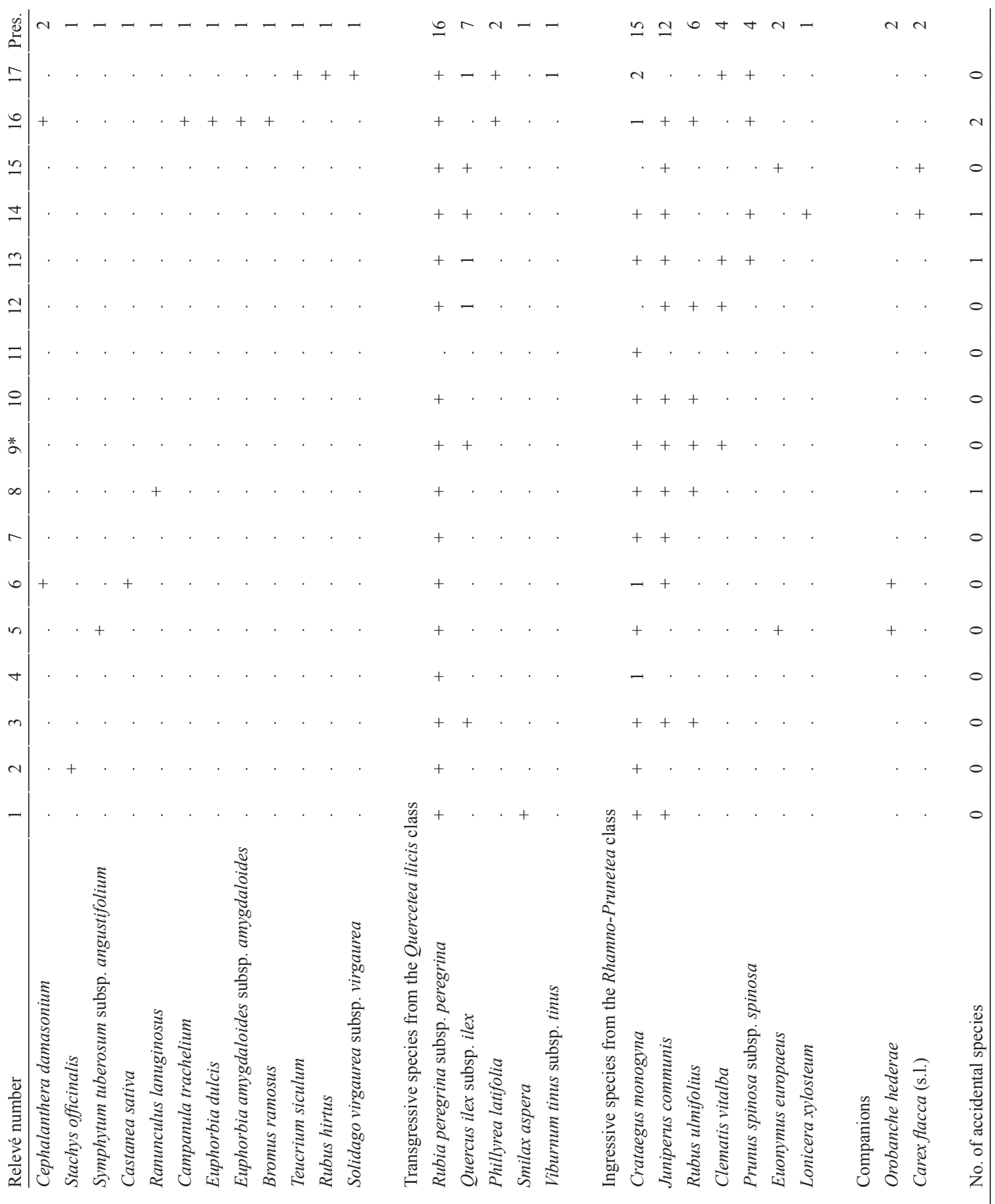

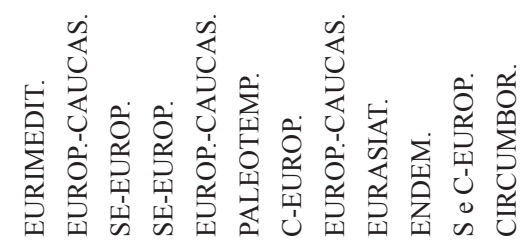
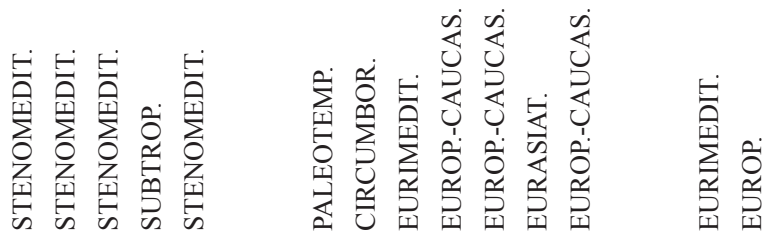

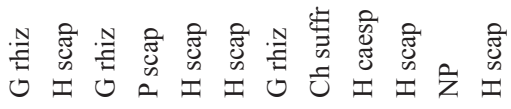

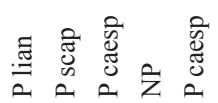

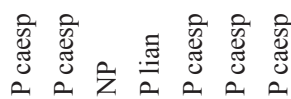

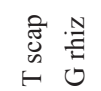


Table 6 (Tabela 6): Malo florentinae-Quercetum frainetto Biondi, Gigante, Pignattelli \& Venanzoni 2001 viburnetosum tini Biondi, Gigante, Pignattelli \& Venanzoni 2001.

\begin{tabular}{|c|c|c|c|c|c|c|c|c|c|c|}
\hline Relevé number & 1 & 2 & 3 & 4 & 5 & 6 & 7 & 8 & \multirow{8}{*}{$\dot{0}$} & \multirow{8}{*}{ 总 } \\
\hline Relevé number in Figure 1 & 73 & 74 & 75 & 76 & 77 & 78 & 79 & 80 & & \\
\hline Altitude (m a.s.1.) & 180 & 160 & 190 & 220 & 220 & 220 & 220 & 220 & & \\
\hline Aspect & $\mathrm{N}$ & $\mathrm{S}$ & ENE & $\mathrm{N}$ & $\mathrm{N}$ & $\mathrm{N}$ & NNW & $\mathrm{N}$ & & \\
\hline Slope $\left({ }^{\circ}\right)$ & 5 & 5 & 5 & 5 & 10 & 10 & 14 & 20 & & \\
\hline Cover $(\%)$ & 99 & 100 & 100 & 80 & 100 & 90 & 95 & 95 & & \\
\hline Relevé area $\left(\mathrm{m}^{2}\right)$ & 300 & 300 & 300 & 100 & 100 & 100 & 400 & 100 & & \\
\hline Morphology & flat & slope & slope & slope & slope & slope & slope & slope & & \\
\hline
\end{tabular}

\begin{tabular}{|c|c|c|c|c|c|c|c|c|c|c|c|c|}
\hline \multirow[b]{2}{*}{$P$ caesp } & \multirow[b]{2}{*}{ CIRCUMBOR. } & \multicolumn{11}{|c|}{ Characteristic species of the Malo florentinae-Quercetum frainetto association } \\
\hline & & Juniperus communis & 2 & 2 & + & + & + & + & + & + & 8 & 100 \\
\hline H caesp & EUROP.-CAUCAS. & Festuca heterophylla & + & + & + & 2 & + & + & . & + & 7 & 88 \\
\hline P caesp & NE-STENOMEDIT. & Malus florentina & 1 & 1 & + & . & . & . & + & . & 4 & 50 \\
\hline H scap & EUROP.-CAUCAS. & Hieracium racemosum & + & + & + & . & . & . & . & . & 3 & 38 \\
\hline \multicolumn{13}{|c|}{ Differential species of the viburnetosum tini subassociation } \\
\hline G rhiz & STENOMEDIT. & Asparagus acutifolius & 1 & 1 & + & + & 2 & 1 & 1 & + & 8 & 100 \\
\hline P scap & STENOMEDIT. & Quercus ilex subsp. ilex & + & 1 & + & 2 & . & . & 1 & 2 & 6 & 75 \\
\hline NP & EURIMEDIT. & Osyris alba & 2 & 2 & + & 1 & . & . & 1 & . & 5 & 63 \\
\hline$P$ caesp & STENOMEDIT. & Viburnum tinus subsp. tinus & + & + & + & . & 1 & . & . & . & 4 & 50 \\
\hline
\end{tabular}

Characteristic and differential species of the Crataego laevigatae-Quercenion cerridis suballiance and of the Crataego laevigatae-Quercion cerridis alliance

\begin{tabular}{|c|c|c|c|c|c|c|c|c|c|c|c|c|}
\hline NP & EUROP.-CAUCAS. & Ligustrum vulgare & 1 & 2 & 2 & + & 2 & 2 & 1 & 1 & 8 & 100 \\
\hline G rhiz & EUROP.-CAUCAS. & Lathyrus niger & + & + & + & + & + & + & . & . & 6 & 75 \\
\hline $\mathrm{H}$ ros & EURIMEDIT. & Potentilla micrantha & + & + & + & + & . & . & . & 1 & 5 & 63 \\
\hline H scap & EUROP.-CAUCAS. & Stachys officinalis & + & + & + & + & . & . & . & + & 5 & 63 \\
\hline H scap & EURIMEDIT.-SUBATL. & Oenanthe pimpinelloides & + & 1 & + & + & . & . & . & . & 4 & 50 \\
\hline H scap & EUROSIBER. & Serratula tinctoria subsp. tinctoria & 1 & + & + & . & . & . & . & . & 3 & 38 \\
\hline H caesp & EURIMEDIT. & Poa sylvicola & + & 1 & + & . & . & . & . & . & 3 & 38 \\
\hline P scap & EURASIAT. & Pyrus communis & . & 1 & + & . & . & . & . & . & 2 & 25 \\
\hline H scap & ENDEM. & Teucrium siculum & . & . & + & + & . & . & . & . & 2 & 25 \\
\hline H rept & EURASIAT. & Veronica officinalis & . & + & . & . & . & . & . & . & 1 & 13 \\
\hline $\mathrm{H}$ ros & S-EUROP.-SUDSIBER. & Silene viridiflora & . & . & + & . & . & . & . & . & 1 & 13 \\
\hline$P$ caesp & STENOMEDIT. & Pyracantha coccinea & . & . & + & . & . & . & . & . & 1 & 13 \\
\hline
\end{tabular}

\begin{tabular}{|c|c|c|c|c|c|c|c|c|c|c|c|}
\hline P scap & N-EURIMEDIT. & Quercus cerris & 1 & 2 & 2 & . & 4 & 4 & 2 & 1 & 7 \\
\hline P caesp & PALEOTEMP. & Sorbus torminalis & + & . & 1 & + & 1 & 1 & + & + & 7 \\
\hline NP & C-EUROP. & Emerus majus subsp. emeroides & . & + & + & + & 1 & 1 & 2 & + & 7 \\
\hline H scap & PONTICA & Buglossoides purpurocaerulea & + & 1 & 1 & . & + & + & 1 & . & 6 \\
\hline P scap & EURIMEDIT. & Sorbus domestica & + & + & 1 & . & + & + & + & . & 6 \\
\hline P caesp & S-EUROP.-SUDSIBER. & Cornus mas & . & . & + & + & 2 & 2 & + & + & 6 \\
\hline H scap & SUBCOSMOP. & Agrimonia eupatoria subsp. eupatoria & + & + & + & 1 & . & . & . & . & 4 \\
\hline $\mathrm{H}$ ros & EURIMEDIT. & Viola alba subsp. dehnhardtii & + & + & + & . & . & . & + & . & 4 \\
\hline G bulb & NW-STENOMEDIT. & Cyclamen repandum subsp. repandum & + & + & + & . & . & + & . & . & 4 \\
\hline P caesp & SE-EUROP. & Quercus pubescens (s.1.) & . & + & 1 & . & . & . & . & . & 2 \\
\hline Ch suffr & SUBATL. & Helleborus foetidus & . & + & + & . & . & . & . & . & 2 \\
\hline
\end{tabular}




\begin{tabular}{|c|c|c|c|c|c|c|c|c|c|c|c|c|}
\hline & & Relevé number & 1 & 2 & 3 & 4 & 5 & 6 & 7 & 8 & Freq. & Freq. \\
\hline $\mathrm{H}$ ros & EURIMEDIT. & Silene italica subsp. italica & . & + & . & . & . & . & . & . & 1 & 13 \\
\hline H caesp & EUROP.-CAUCAS. & Hypericum montanum & . & . & + & . & . & . & . & . & 1 & 13 \\
\hline \multirow[t]{2}{*}{ P scap } & EUROP. & Quercus petraea & . & . & . & + & . & . & . & . & 1 & 13 \\
\hline & & Characteristic species of the Querco-Fagetea cl & & & & & & & & & & \\
\hline P scap & SE-EUROP. & Quercus frainetto & 4 & 3 & 2 & 4 & 3 & 3 & 4 & 4 & 8 & 100 \\
\hline Ch frut & EURIMEDIT. & Ruscus aculeatus & 3 & 1 & 1 & 2 & 3 & 3 & 2 & 2 & 8 & 100 \\
\hline P lian & EURIMEDIT. & Hedera helix subsp. helix & 2 & 2 & + & 1 & 3 & 3 & 1 & 1 & 8 & 100 \\
\hline G rad & EURIMEDIT. & Tamus communis & + & + & + & 1 & 1 & 1 & 1 & 1 & 8 & 100 \\
\hline P scap & S-EUROP.-SUDSIBER. & Fraxinus ornus subsp. ornus & 1 & 2 & 1 & . & + & + & + & 2 & 7 & 88 \\
\hline P scap & EUROP.-CAUCAS. & Acer campestre & . & 1 & 1 & + & 1 & 1 & + & 1 & 7 & 88 \\
\hline G bulb & N-STENOMEDIT. & Cyclamen hederifolium subsp. hederifolium & 1 & + & 1 & + & + & . & . & . & 5 & 63 \\
\hline H caesp & PALEOTEMP. & Brachypodium sylvaticum subsp. sylvaticum & 1 & + & + & 2 & . & . & . & . & 4 & 50 \\
\hline Ch suffr & EUROP.-CAUCAS. & Euphorbia amygdaloides subsp. amygdaloides & + & . & + & + & . & . & 1 & . & 4 & 50 \\
\hline$P$ caesp & EUROP.-CAUCAS. & Ulmus minor subsp. minor & . & + & + & . & 1 & 1 & . & . & 4 & 50 \\
\hline NP & S-MEDIT.-SUBATL. & Rosa arvensis & . & . & + & . & + & . & + & + & 4 & 50 \\
\hline P lian & S-EUROP.-SUDSIBER. & Lonicera caprifolium & + & 1 & 1 & . & . & . & . & . & 3 & 38 \\
\hline H rept & EUROP.-CAUCAS. & Ajuga reptans & + & + & + & . & . & . & . & . & 3 & 38 \\
\hline H scap & EUROSIBER. & Viola reichenbachiana & + & . & + & 1 & . & . & . & . & 3 & 38 \\
\hline H caesp & PALEOTEMP. & Melica uniflora & . & + & 1 & 1 & . & . & . & . & 3 & 38 \\
\hline H scap & CIRCUMBOR. & Geum urbanum & + & + & . & . & . & . & . & . & 2 & 25 \\
\hline P scap & PONTICA & Prunus avium & . & + & . & + & . & . & . & . & 2 & 25 \\
\hline G rhiz & S-EUROP.-SUDSIBER. & Lathyrus venetus & . & . & + & . & . & . & + & . & 2 & 25 \\
\hline G rhiz & EURIMEDIT. & Cephalanthera damasonium & + & . & . & . & . & . & . & . & 1 & 13 \\
\hline H caesp & EURIMEDIT. & Luzula forsteri & + & . & . & . & . & . & . & . & 1 & 13 \\
\hline H rept & EUROSIB. & Asarum europaeum & + & . & . & . & . & . & . & . & 1 & 13 \\
\hline Ch suffr & EURASIAT. & Genista tinctoria & . & + & . & . & . & . & . & . & 1 & 13 \\
\hline G bulb & EUROSIB. & Platanthera chlorantha & . & + & . & . & . & . & . & . & 1 & 13 \\
\hline G rhiz & PALEOTEMP. & Epipactis helleborine subsp. helleborine & . & + & . & . & . & . & . & . & 1 & 13 \\
\hline H scap & EUROP. & Viola riviniana & . & . & + & . & . & . & . & . & 1 & 13 \\
\hline H caesp & SUBATL. & Brachypodium rupestre subsp. rupestre & . & . & . & . & . & . & 2 & . & 1 & 13 \\
\hline $\mathrm{H}$ ros & EUROP.-CAUCAS. & Primula vulgaris & . & . & . & . & . & . & + & . & 1 & 13 \\
\hline Ch rept & EUROP.-CAUCAS. & Vinca minor & . & . & . & . & . & . & . & 3 & 1 & 13 \\
\hline
\end{tabular}

$\begin{array}{ll}\text { P lian } & \text { STENOMEDIT. } \\ \text { NP } & \text { STENOMEDIT. } \\ \text { P caesp } & \text { STENOMEDIT. } \\ \text { NP } & \text { SUBTROP. } \\ \text { P caesp } & \text { STENOMEDIT. } \\ \text { P caesp } & \text { EURIMEDIT. }\end{array}$

$\begin{array}{ll}\text { P caesp } & \text { C-EUROP. } \\ P \text { caesp } & \text { EUROP.-CAUCAS. } \\ P \text { caesp } & \text { EURASIAT. } \\ P \text { caesp } & \text { PALEOTEMP. } \\ P \text { caesp } & \text { EURASIAT. } \\ P \text { caesp } & \text { OROF. SW-EUROP. } \\ P \text { lian } & \text { EURIMEDIT. }\end{array}$

Transgressive species from the Quercetea ilicis class

Rubia peregrina subsp. peregrina

Rosa sempervirens

Erica arborea

Smilax aspera

Arbutus unedo

Juniperus oxycedrus subsp. oxycedrus

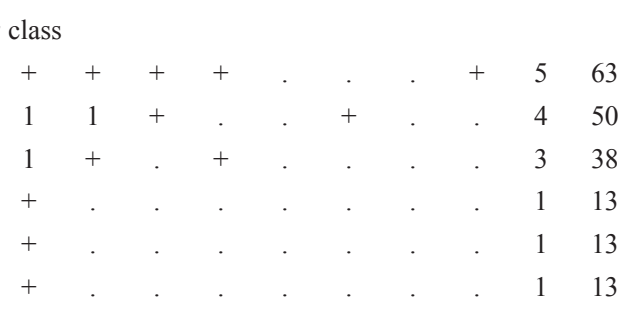

Ingressive species from the Rhamno-Prunetea class

Crataegus laevigata

Prunus spinosa subsp. spinosa

Euonymus europaeus

Crataegus monogyna

Cornus sanguinea (s.1.)

Cytisophyllum sessilifolium

Lonicera etrusca

$\begin{array}{cccccccccc}+ & 1 & + & . & + & + & + & + & 7 & 88 \\ + & 1 & + & . & 1 & 1 & + & . & 6 & 75 \\ + & + & + & \cdot & + & + & . & . & 5 & 63 \\ . & . & 1 & + & + & + & + & \cdot & 5 & 63 \\ + & 1 & 2 & . & . & \cdot & + & . & 4 & 50 \\ . & \cdot & + & . & + & + & 2 & . & 4 & 50 \\ . & . & . & 1 & . & . & + & 2 & 3 & 38\end{array}$




\begin{tabular}{|c|c|c|c|c|c|c|c|c|c|c|c|c|}
\hline & & Relevé number & 1 & 2 & 3 & 4 & 5 & 6 & 7 & 8 & Freq. & Freq. \\
\hline NP & S-EUROP.-SUDSIB. & Rosa gallica & + & + & $\cdot$ & . & . & . & $\cdot$ & . & 2 & 25 \\
\hline NP & PALEOTEMP. & Rosa canina (s.1.) & . & + & . & . & . & . & . & . & 1 & 13 \\
\hline$P$ caesp & EUROSIBER. & Prunus mahaleb & . & . & . & 2 & . & . & . & . & 1 & 13 \\
\hline NP & EURIMEDIT. & Rubus ulmifolius & . & . & . & . & . & . & + & . & 1 & 13 \\
\hline \multirow[t]{2}{*}{$P$ caesp } & STENOMEDIT. & Pyracantha coccinea & . & . & . & . & . & . & + & . & 1 & 13 \\
\hline & & Companions & & & & & & & & & & \\
\hline G rhiz & EUROP. & Carex flacca (s.1.) & + & 1 & + & . & . & . & . & $\cdot$ & 3 & 38 \\
\hline H scap & EUROP.-CAUCAS. & Calamintha nepeta subsp. sylvatica & + & + & + & . & . & . & . & . & 3 & 38 \\
\hline Ch suffr & C-EUROP. & Genista germanica & + & + & . & + & . & . & . & . & 3 & 38 \\
\hline H scap & SE-EUROP. & Centaurea jacea subsp. gaudini & + & + & . & . & . & . & . & . & 2 & 25 \\
\hline T scap & EURASIAT. & Melampyrum cristatum subsp. cristatum & + & + & . & . & . & . & . & . & 2 & 25 \\
\hline H scap & EUROP.-CAUCAS. & Inula salicina & + & + & . & . & . & . & . & . & 2 & 25 \\
\hline H scap & EUROSIBER. & Peucedanum cervaria & + & + & . & . & . & . & . & . & 2 & 25 \\
\hline H caesp & EURIMEDIT. & Phleum bertolonii & + & + & . & . & . & . & . & . & 2 & 25 \\
\hline H scap & CIRCUMBOR. & Prunella vulgaris & + & . & + & . & . & . & . & . & 2 & 25 \\
\hline H scap & EURASIAT. & Cruciata laevipes & + & . & + & . & . & . & . & . & 2 & 25 \\
\hline P scap & AVV. NATUR. & Robinia pseudacacia & . & + & + & . & . & . & . & . & 2 & 25 \\
\hline Ch frut & CIRCUMBOR. & Calluna vulgaris & + & . & . & . & . & . & . & . & 1 & 13 \\
\hline H scap & CIRCUMBOR. & Clinopodium vulgare subsp. vulgare & + & . & . & . & . & . & . & . & 1 & 13 \\
\hline Ch suffr & EURIMEDIT. & Dorycnium hirsutum & + & . & . & . & . & . & . & . & 1 & 13 \\
\hline H bienne & EUROP.-CAUCAS. & Inula conyzae & . & + & . & . & . & . & . & . & 1 & 13 \\
\hline H scap & SE-EUROP. & Ptilostemon strictus & . & + & . & . & . & . & . & . & 1 & 13 \\
\hline G bulb & W-MEDIT.-MONT. & Colchicum lusitanum & . & . & + & . & . & . & . & . & 1 & 13 \\
\hline H scand & EUROP.-CAUCAS. & Lathyrus sylvestris & . & . & + & . & . & . & . & . & 1 & 13 \\
\hline G rhiz & STENOMEDIT. & Arum italicum & . & . & . & . & . & + & . & . & 1 & 13 \\
\hline Ch suffr & EURIMEDIT. & Teucrium chamaedrys subsp. chamaedrys & . & . & . & . & . & . & + & . & 1 & 13 \\
\hline H scap & EUROP.-CAUCAS. & Geranium sanguineum & . & . & . & . & . & . & + & . & 1 & 13 \\
\hline
\end{tabular}




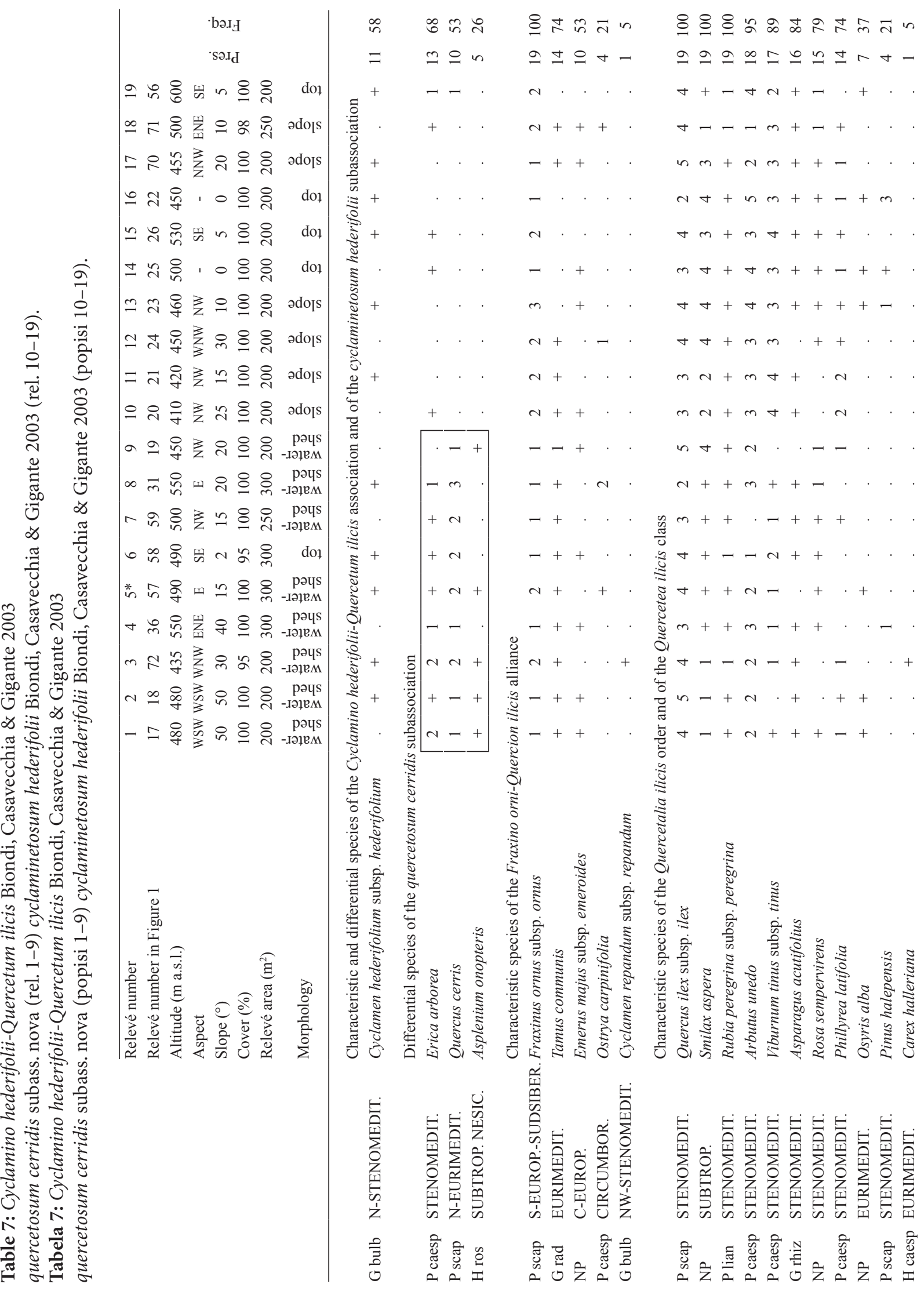




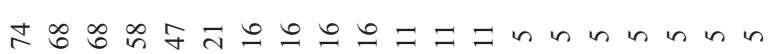

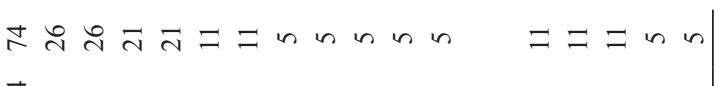

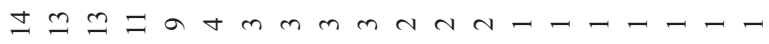

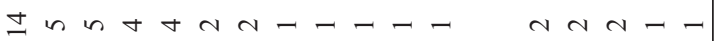

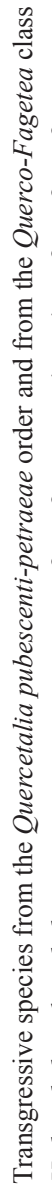

$\mathrm{Na}+\mathrm{N}$
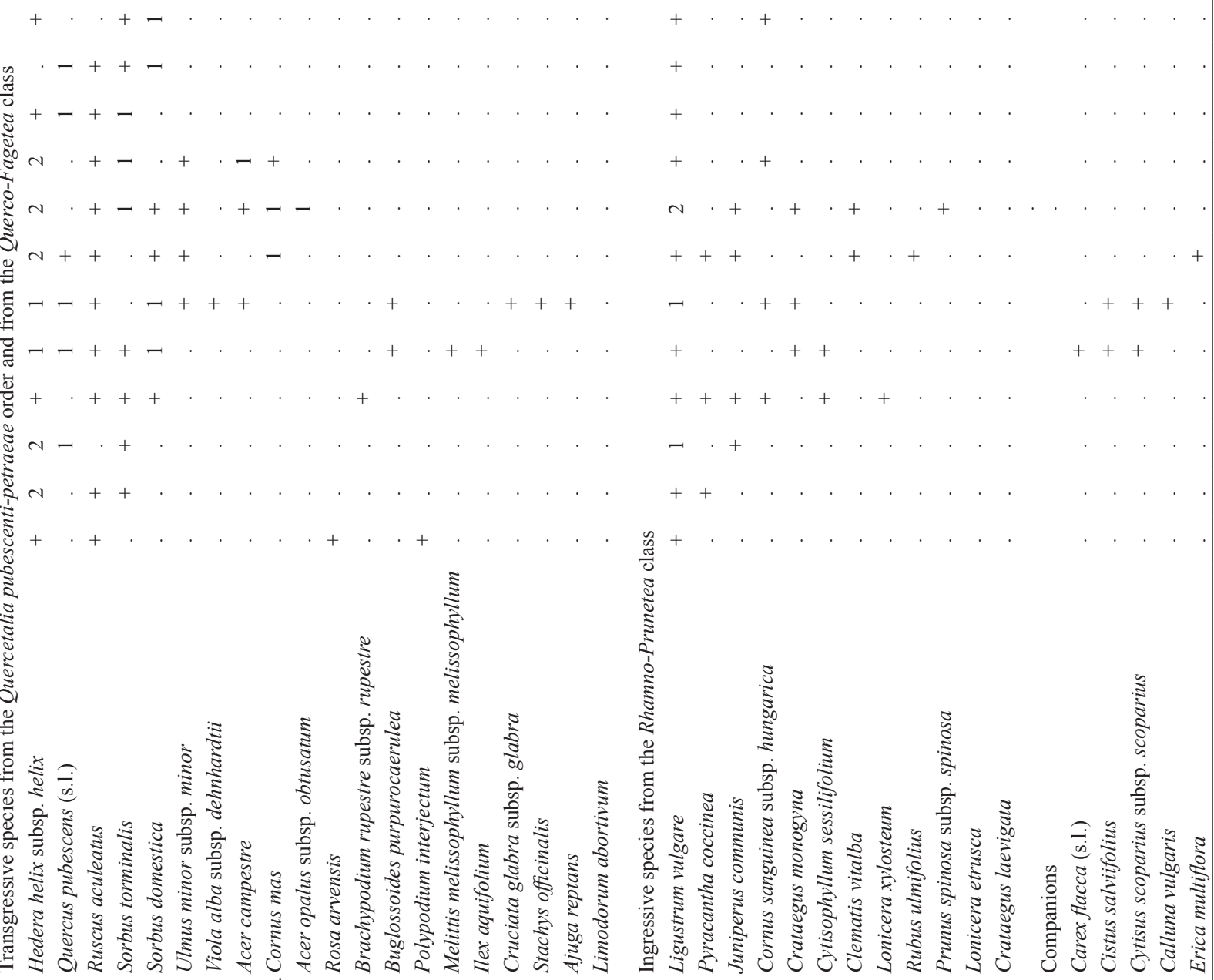

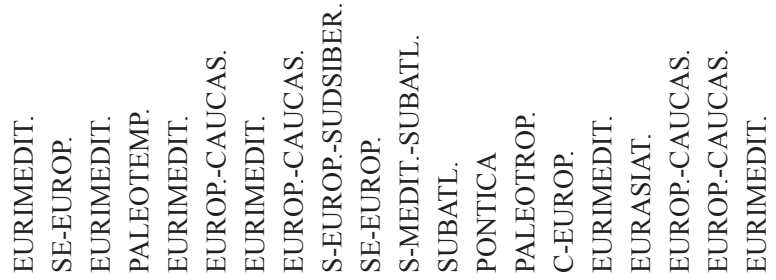

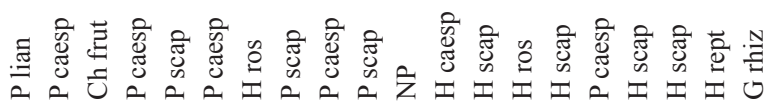

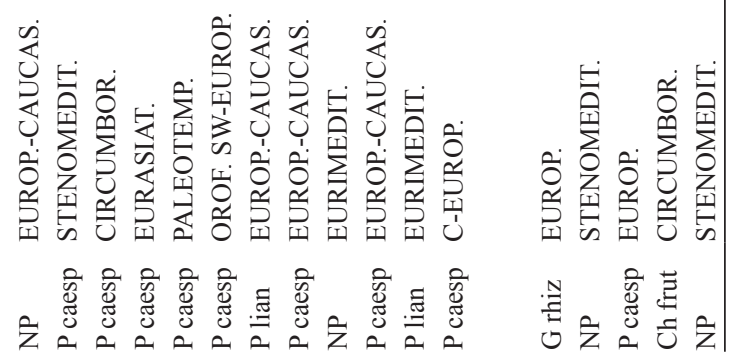


Table 8: Synoptical scheme of holm-oak woods referred to Arbuto unedonis-Quercetum ilicis and Cyclamino hederifolii-Quercetum ilicis cyclaminetosum hederifolii, compared with those of the study area. Column 1 - Arbuto unedonis-Quercetum ilicis - Table 14 in Di Pietro et al. 2010; column 2 - Cyclamino hederifolii-Quercetum ilicis cyclaminetosum hederifolii - Table 3, rel. 1-15 in Biondi et al. 2003; column 3 - cluster IIa ${ }^{\mathrm{I}}$, present study; column 4 - cluster IIa ${ }^{\mathrm{II}}$, present study.

Tabela 8: Sinoptična shema gozdov črničevja asociacij Arbuto unedonis-Quercetum ilicis in Cyclamino hederifolii-Quercetum ilicis cyclaminetosum hederifolii, primerjani s tistimi v obravnavanem območju. Stolpec $1-$ Arbuto unedonis-Quercetum ilicis - Tabela $14 \mathrm{v}$ Di Pietro et al. 2010; stolpec 2 - Cyclamino hederifolii-Quercetum ilicis cyclaminetosum hederifolii - Table 3, popisi 1-15 v Biondi et al. 2003; stolpec 3 - klaster IIa ${ }^{\mathrm{I}}$, ta članek; stolpec 4 - klaster IIa ${ }^{\mathrm{II}}$, ta članek.

\begin{tabular}{l} 
Column nr. \\
Nr. of relevés \\
\hline Characteristic species of Quercetea ilicis class \\
Quercus ilex subsp. ilex \\
Rubia peregrina subsp. peregrina \\
Smilax aspera \\
Phillyrea latifolia \\
Asplenium onopteris \\
Arbutus unedo \\
Viburnum tinus subsp. tinus \\
Erica arborea \\
Asparagus acutifolius \\
Carex distachya \\
Laurus nobilis \\
Pistacia lentiscus \\
Rosa sempervirens \\
Osyris alba \\
Carex halleriana \\
Pinus halepensis \\
Myrtus communis \\
Rhamnus alaternus subsp. alaternus \\
Clematis flammula \\
Arisarum vulgare \\
Pistacia terebinthus subsp. terebinthus \\
Ampelodesmos mauritanicus \\
Lonicera implexa subsp. implexa \\
Pulicaria odora \\
Calicotome spinosa \\
The
\end{tabular}

Transgressive species from the Querco-Fagetea class

Fraxinus ornus subsp. ornus

Tamus communis

Ruscus aculeatus

Quercus pubescens (s.l.)

Cyclamen repandum subsp. repandum

Hedera helix subsp. helix

Viola alba subsp. dehnhardtii

Sorbus domestica

Ostrya carpinifolia

Melica uniflora

\begin{tabular}{cccc}
1 & 2 & 3 & 4 \\
16 & 15 & 9 & 10 \\
\hline
\end{tabular}

$\begin{array}{lllll}\mathrm{V} & \mathrm{V} & \mathrm{V} & \mathrm{V}\end{array}$

$\begin{array}{llll}\mathrm{V} & \mathrm{V} & \mathrm{V} & \mathrm{V}\end{array}$

$\begin{array}{llll}\mathrm{V} & \mathrm{V} & \mathrm{V} & \mathrm{V}\end{array}$

$\mathrm{V} \quad \mathrm{V}$ IV $\mathrm{V}$

$\begin{array}{llll}\mathrm{V} & \text { II } & \text { II } & \text { I }\end{array}$

IV IV $\mathrm{V}$ V

III IV IV V

III I $\mathrm{V}$ IV

II $\mathrm{V}$ IV $\mathrm{V}$

II I I .

I I . I

. III I I

. II $\mathrm{V}$ IV

. I III II

. I I I

. I I I

II II .

. III .

. III .

. II .

. I .

. I .

. I .

$\begin{array}{lll}\cdot & \mathrm{I} & \cdot \\ . & \mathrm{I} & . \\ . & \mathrm{I}\end{array}$

\section{sus}

$\mathrm{V}$ IV IV IV

$\mathrm{V}$ IV IV III

$\mathrm{V}$ II IV IV

$\begin{array}{llll}\mathrm{V} & \mathrm{I} & \mathrm{I} & \mathrm{I}\end{array}$

II II IV IV

II II II I

II I III III

I II I I

I I . I

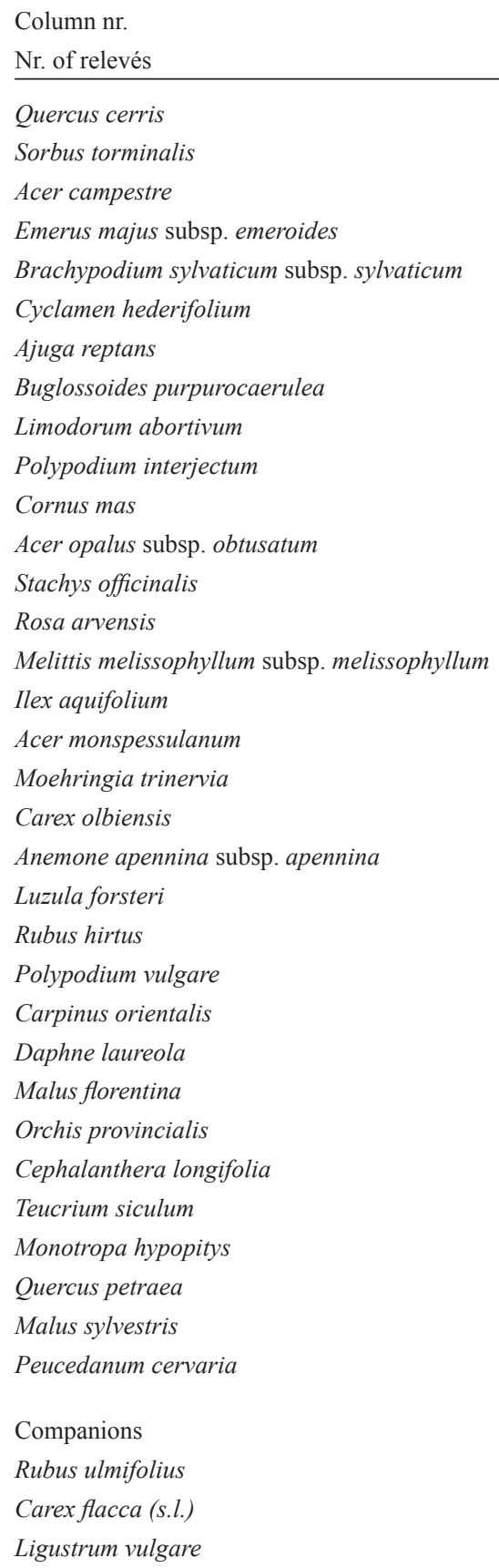

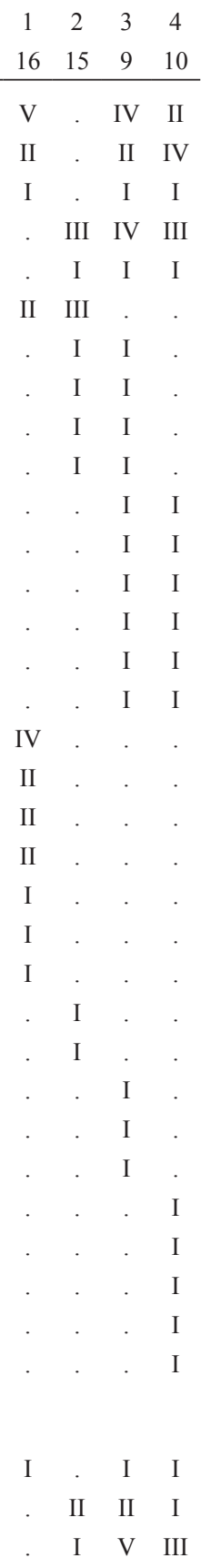




\begin{tabular}{|c|c|c|c|c|c|c|c|c|c|}
\hline Column nr. & 1 & 2 & 3 & 4 & Column nr. & 1 & 2 & 3 & 4 \\
\hline Nr. of relevés & 16 & 15 & 9 & 10 & Nr. of relevés & 16 & 15 & 9 & 10 \\
\hline Brachypodium pinnatum/rupestre & . & I & II & I & Juniperus communis & . & . & III & I \\
\hline Lonicera etrusca & . & I & I & I & Pyracantha coccinea & . & . & III & I \\
\hline Juniperus oxycedrus subsp. oxycedrus & . & I & I & I & Ulmus minor subsp. minor & . & . & II & I \\
\hline Crataegus monogyna & . & I & I & I & Cytisophyllum sessilifolium & . & . & I & I \\
\hline Clematis vitalba & . & I & I & I & Cruciata glabra subsp. glabra & . & . & I & I \\
\hline Cornus sanguinea (s.l.) & . & I & I & I & Erica multiflora & . & . & I & I \\
\hline Geranium purpureum & II & I & . & . & Veronica officinalis & . & . & I & I \\
\hline Oryzopsis miliacea & I & I & . & . & & & & & \\
\hline
\end{tabular}

Table 9: Roso sempervirentis-Quercetum pubescentis Biondi 1986 ericetosum multiflorae Catorci \& Orsomando 1997 (rel. 1-6) quercetosum pubescentis Allegrezza, Baldoni, Biondi, Taffetani \& Zuccarello 2002 (rel. 7-10)

Tabela 9: Roso sempervirentis-Quercetum pubescentis Biondi 1986 ericetosum multiflorae Catorci \& Orsomando 1997 (popisi 1-6) quercetosum pubescentis Allegrezza, Baldoni, Biondi, Taffetani \& Zuccarello 2002 (popisi 7-10)

\begin{tabular}{|c|c|c|c|c|c|c|c|c|c|c|c|c|}
\hline Relevé number & 1 & 2 & 3 & 4 & 5 & 6 & 7 & 8 & 9 & 10 & \multirow{8}{*}{$\dot{\mathscr{D}}$} & \multirow{8}{*}{ 远 } \\
\hline Relevé number in Fig. 1 & 60 & 62 & 64 & 61 & 66 & 63 & 65 & 68 & 67 & 69 & & \\
\hline Altitude (m a.s.l.) & 390 & 400 & 460 & 450 & 380 & 410 & 430 & 300 & 300 & 550 & & \\
\hline Aspect & SE & S & SSW & SSW & S & SSE & SSE & SSE & SW & $\mathrm{S}$ & & \\
\hline Slope $\left(^{\circ}\right)$ & 40 & 5 & 20 & 20 & 30 & 15 & 25 & 25 & 40 & 30 & & \\
\hline Cover $(\%)$ & 95 & 98 & 90 & 90 & 95 & 95 & 95 & 95 & 95 & 95 & & \\
\hline Relevé area $\left(\mathrm{m}^{2}\right)$ & 150 & 150 & 150 & 200 & 200 & 150 & 200 & 150 & 150 & 200 & & \\
\hline Morphology & $\frac{0}{\frac{0}{n}}$ & $\frac{\tilde{a}}{\tilde{n}}$ & $\frac{\tilde{O}}{n}$ & $\frac{\tilde{O}}{n}$ & $\frac{\tilde{a}}{\frac{0}{n}}$ & $\frac{\tilde{a}}{n}$ & $\frac{\tilde{a}}{\tilde{n}}$ & $\frac{\tilde{0}}{n}$ & $\frac{\tilde{a}}{n}$ & $\frac{\tilde{a}}{n}$ & & \\
\hline
\end{tabular}

\begin{tabular}{|c|c|c|c|c|c|c|c|c|c|c|c|c|c|c|}
\hline \multirow[b]{2}{*}{ P lian } & \multirow[b]{2}{*}{ STENOMEDIT. } & \multicolumn{13}{|c|}{ Characteristic and differential species of the Roso sempervirentis-Quercetum pubescentis association } \\
\hline & & Rubia peregrina subsp. peregrina & 1 & 2 & 2 & 2 & 1 & 1 & 2 & 3 & 1 & 2 & 10 & 100 \\
\hline NP & STENOMEDIT. & Rosa sempervirens & 1 & 1 & 1 & 1 & 1 & 1 & 1 & 1 & 1 & 1 & 10 & 100 \\
\hline $\mathrm{P}$ lian & EURIMEDIT. & Lonicera etrusca & + & + & 1 & + & + & + & + & 1 & + & 1 & 10 & 100 \\
\hline P lian & EURIMEDIT. & Clematis flammula & . & + & 1 & + & . & 1 & + & + & . & . & 6 & 60 \\
\hline P lian & STENOMEDIT. & Lonicera implexa & . & . & . & + & + & + & . & . & . & . & 3 & 30 \\
\hline \multirow[t]{2}{*}{ NP } & SUBTROP. & Smilax aspera & . & . & . & . & . & . & . & + & + & + & 3 & 30 \\
\hline & & \multicolumn{7}{|c|}{ Differential species of the ericetosum multiflorae subassociation } & & & & & & \\
\hline P caesp & STENOMEDIT. & Pyracantha coccinea & + & + & 3 & 2 & + & + & 1 & . & + & . & 8 & 80 \\
\hline P scap & STENOMEDIT. & Pinus halepensis & 2 & 2 & 2 & 3 & 2 & 2 & 1 & . & . & . & 7 & 70 \\
\hline \multirow[t]{2}{*}{ NP } & STENOMEDIT. & Erica multiflora & 1 & 1 & 2 & 2 & 1 & 1 & + & . & . & . & 7 & 70 \\
\hline & & \multicolumn{13}{|c|}{$\begin{array}{l}\text { Characteristic species of the Lauro nobilis-Quercenion pubescentis suballiance and of the Carpinion orientalis } \\
\text { alliance }\end{array}$} \\
\hline G rhiz & STENOMEDIT. & Asparagus acutifolius & 1 & 1 & 1 & 1 & 1 & 1 & 1 & 2 & + & 2 & 10 & 100 \\
\hline$P$ caesp & CIRCUMBOR. & Ostrya carpinifolia & 1 & + & . & + & . & + & . & . & + & + & 6 & 60 \\
\hline NP & C-EUROP. & Emerus majus subsp. emeroides & + & + & + & . & . & . & . & + & + & . & 5 & 50 \\
\hline H caesp & SE-EUROP. & Sesleria autumnalis & + & . & + & . & + & . & . & + & . & . & 4 & 40 \\
\hline H scap & SE-EUROP. & Cnidium silaifolium & . & . & . & + & + & + & . & . & + & . & 4 & 40 \\
\hline P scap & SE-EUROP. & Acer opalus subsp. obtusatum & + & . & . & . & . & + & . & . & . & . & 2 & 20 \\
\hline P scap & S-EUROP.-SUDSIBER. & Cercis siliquastrum & . & . & . & . & . & + & . & . & . & + & 2 & 20 \\
\hline P caesp & EURIMEDIT. & Pistacia terebinthus subsp. terebinthus & . & . & . & . & . & . & . & . & + & . & 1 & 10 \\
\hline
\end{tabular}


Characteristic species of the Quercetalia pubescenti-petraeae order

\begin{tabular}{|c|c|c|c|c|c|c|c|c|c|c|c|c|c|c|}
\hline P caesp & SE-EUROP. & Quercus pubescens (s.1.) & 2 & 2 & 1 & 1 & 2 & 2 & 3 & 4 & 4 & 4 & 10 & 100 \\
\hline H caesp & SUBATL. & Brachypodium rupestre subsp. rupestre & 1 & 1 & 2 & 1 & 2 & 2 & 1 & 1 & 4 & 2 & 10 & 100 \\
\hline P scap & S-EUROP.-SUDSIBER. & Fraxinus ornus subsp. ornus & + & 1 & 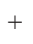 & + & + & 1 & 1 & 2 & 1 & + & 10 & 100 \\
\hline H scap & PONTICA & Buglossoides purpurocaerulea & + & + & + & . & + & 1 & + & 1 & + & + & 9 & 90 \\
\hline P scap & EURIMEDIT. & Sorbus domestica & . & + & + & 1 & . & + & 1 & 1 & + & 1 & 8 & 80 \\
\hline $\mathrm{H}$ ros & EURIMEDIT. & Viola alba subsp. dehnhardtii & + & + & + & + & . & + & . & $\cdot$ & + & + & 7 & 70 \\
\hline P caesp & PALEOTEMP. & Sorbus torminalis & 1 & + & 2 & + & . & + & + & $\cdot$ & . & + & 7 & 70 \\
\hline P caesp & EURIMEDIT. & Acer monspessulanum & 1 & . & + & . & . & $\cdot$ & + & + & 2 & 1 & 6 & 60 \\
\hline G rhiz & EURIMEDIT. & Limodorum abortivum & $\cdot$ & . & + & + & . & + & . & $\cdot$ & . & . & 3 & 30 \\
\hline Ch suffr & SUBATL. & Helleborus foetidus & $\cdot$ & . & . & . & . & . & . & + & + & . & 2 & 20 \\
\hline H scap & NE-MEDIT.-MONT. & Scutellaria columnae subsp. columnae & $\cdot$ & . & . & . & . & $\cdot$ & . & + & . & . & 1 & 10 \\
\hline P scap & N-EURIMEDIT. & Quercus cerris & . & . & . & . & . & . & . & + & . & . & 1 & 10 \\
\hline
\end{tabular}

$\begin{array}{lll}\text { G rad } & \text { EURIMEDIT. } & \text { Tamus communis } \\ \text { P scap } & \text { EUROP.CAUCAS. } & \text { Acer campestre } \\ \text { P lian } & \text { EURIMEDIT. } & \text { Hedera helix subsp. helix } \\ \text { P scap } & \text { PONTICA } & \text { Prunus avium } \\ \text { G rhiz } & \text { EURIMEDIT. } & \text { Cephalanthera damasonium } \\ \text { G rhiz } & \text { EURASIAT. } & \text { Cephalanthera rubra } \\ \text { G rhiz } & \text { EURASIAT. } & \text { Cephalanthera longifolia } \\ \text { Ch frut } & \text { EURIMEDIT. } & \text { Ruscus aculeatus }\end{array}$

Transgressive species from the Quercetea ilicis class

\begin{tabular}{|c|c|c|c|c|c|c|c|c|c|c|c|c|c|c|}
\hline$P$ caesp & STENOMEDIT. & Arbutus unedo & 2 & 2 & 2 & + & 1 & 2 & 1 & 1 & 1 & + & 10 & 100 \\
\hline P caesp & EURIMEDIT. & Juniperus oxycedrus subsp. oxycedrus & . & + & 1 & 1 & 1 & 1 & + & + & + & 1 & 9 & 90 \\
\hline NP & EURIMEDIT. & Osyris alba & 1 & 1 & + & + & 1 & 1 & . & + & + & $\cdot$ & 8 & 80 \\
\hline P caesp & STENOMEDIT. & Viburnum tinus subsp. tinus & 3 & 1 & + & . & 1 & + & 1 & + & + & $\cdot$ & 8 & 80 \\
\hline P caesp & STENOMEDIT. & Phillyrea latifolia & . & . & . & . & . & 2 & + & . & + & . & 3 & 30 \\
\hline
\end{tabular}

\begin{tabular}{|c|c|c|c|c|c|c|c|c|c|c|c|c|c|c|}
\hline P caesp & EURASIAT. & Cornus sanguinea subsp. hungarica & 1 & + & 1 & + & + & 1 & 1 & 1 & + & + & 10 & 100 \\
\hline NP & EUROP.-CAUCAS. & Ligustrum vulgare & + & + & 1 & + & 1 & 1 & 1 & + & + & $\cdot$ & 9 & 90 \\
\hline NP & PALEOTEMP. & Rosa canina (s.1.) & + & + & + & + & + & + & + & + & . & + & 9 & 90 \\
\hline$P$ caesp & EURIMEDIT. & Spartium junceum & + & + & 1 & 1 & + & + & . & $\cdot$ & . & + & 7 & 70 \\
\hline P caesp & CIRCUMBOR. & Juniperus communis & + & + & 1 & + & $\cdot$ & + & . & + & . & $\cdot$ & 6 & 60 \\
\hline P lian & EUROP.-CAUCAS. & Clematis vitalba & + & + & + & . & . & + & . & . & + & . & 5 & 50 \\
\hline P caesp & OROF. SW-EUROP. & Cytisophyllum sessilifolium & 1 & . & . & . & . & + & + & . & 1 & + & 5 & 50 \\
\hline NP & EURIMEDIT. & Rubus ulmifolius & + & + & + & + & . & . & . & . & . & $\cdot$ & 4 & 40 \\
\hline$P$ caesp & EUROP.-CAUCAS. & Prunus spinosa subsp. spinosa & . & + & + & . & . & . & + & + & . & . & 4 & 40 \\
\hline P lian & S-EUROP.-SUDSIBER. & Lonicera caprifolium & . & . & . & . & + & . & . & . & . & 1 & 2 & 20 \\
\hline P caesp & PALEOTEMP. & Crataegus monogyna & . & . & . & . & . & . & + & . & + & . & 2 & 20 \\
\hline
\end{tabular}

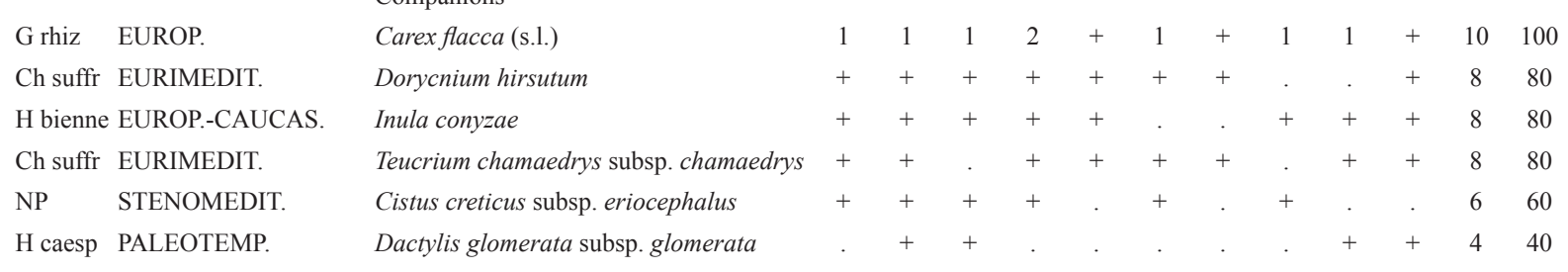




\begin{tabular}{|c|c|c|c|c|c|c|c|c|c|c|c|c|c|c|}
\hline & & Relevé number & 1 & 2 & 3 & 4 & 5 & 6 & 7 & 8 & 9 & 10 & Pres. & Freq. \\
\hline $\mathrm{H}$ caesp & PALEOTEMP. & Bromus erectus subsp. erectus & . & . & + & 1 & . & . & . & . & + & + & 4 & 40 \\
\hline H scand & EUROP.-CAUCAS. & Lathyrus sylvestris & . & + & . & . & . & . & + & + & . & + & 4 & 40 \\
\hline H scap & CIRCUMBOR. & Clinopodium vulgare subsp. vulgare & . & + & . & . & + & . & . & + & . & . & 3 & 30 \\
\hline H bienn & S-EUROP.-SUDSIBER. & Arabis turrita & . & . & . & . & + & . & . & + & + & . & 3 & 30 \\
\hline H scap & EURASIAT. & Cruciata glabra subsp. glabra & . & . & . & . & . & . & . & . & + & + & 2 & 20 \\
\hline $\mathrm{H}$ caesp & COSMOPOL. & Cystopteris fragilis & + & + & . & . & . & . & . & . & . & . & 2 & 20 \\
\hline $\mathrm{H}$ bienne & W-EUROP. & Carduus nutans & . & + & + & . & . & . & . & . & . & . & 2 & 20 \\
\hline Ch suffr & SW-MEDIT.-MONT. & Acinos alpinus subsp. meridionalis & . & . & + & + & . & . & . & . & . & . & 2 & 20 \\
\hline Ch suffr & STENOMEDIT. & Teucrium capitatum subsp. capitatum & . & . & . & + & . & + & . & . & . & . & 2 & 20 \\
\hline H caesp & STENOMEDIT. & Achnatherum bromoides & . & . & . & + & . & + & . & . & . & . & 2 & 20 \\
\hline H scap & SUBCOSMOP. & Agrimonia eupatoria subsp. eupatoria & . & . & . & + & . & . & . & . & . & + & 2 & 20 \\
\hline H scap & EUROSIB. & Picris hieracioides subsp. hieracioides & + & . & . & . & . & . & . & . & . & . & 1 & 10 \\
\hline G rhiz & EUROP.-CAUCAS. & Epipactis sp. & . & + & . & . & . & . & . & . & . & . & 1 & 10 \\
\hline T scap & EURIMEDIT. & Blackstonia perfoliata subsp. perfoliata & . & . & + & . & . & . & . & . & . & . & 1 & 10 \\
\hline
\end{tabular}

Table 10: Ecological features of forest syntaxa (for altitude the whole ranges are indicated, while for slope angle, soil depth, $\mathrm{pH}$ and sand percentage the interquartile ranges are reported).

Tabela 10: Ekološke značilnosti gozdnih sintaksonov (za nadmorsko višino so prikazani celotni razponi, za naklon, globino tal, $\mathrm{pH}$ in odstotek peska pa interkvartilni razpon).

\begin{tabular}{|c|c|c|c|c|c|c|c|c|}
\hline Syntaxon & $\begin{array}{l}\text { Altitude } \\
\text { (m a.s.l.) }\end{array}$ & Geology & Morphology & Aspect & Slope $\left(^{\circ}\right)$ & $\begin{array}{r}\text { Soil depth } \\
(\mathrm{cm})\end{array}$ & Soil pH & Sand $\%$ \\
\hline $\begin{array}{l}\text { Erico arboreae- } \\
\text { Quercetum cerridis } \\
\text { typicum }\end{array}$ & $500-650$ & $\begin{array}{l}\text { Marly-arenaceous } \\
\text { Formation } \\
\text { (sandstone) }\end{array}$ & $\begin{array}{c}\text { Slope, } \\
\text { watershed, top }\end{array}$ & $\begin{array}{l}\text { Southern } \\
\text { (Northern) }\end{array}$ & $5.0-10.0$ & $31.3-35.0$ & $6.1-6.4$ & $77.3-82.3$ \\
\hline $\begin{array}{l}\text { Erico arboreae- } \\
\text { Quercetum cerridis } \\
\text { lathyretosum veneti }\end{array}$ & $450-500$ & $\begin{array}{l}\text { Marly-arenaceous } \\
\text { Formation } \\
\text { (sandstone) }\end{array}$ & Impluvium & Southern & $10.0-15.0$ & $30.0-58.0$ & $6.0-6.3$ & $76.7-80.4$ \\
\hline $\begin{array}{l}\text { Cephalanthero } \\
\text { longifoliae-Quercetum } \\
\text { cerridis }\end{array}$ & $500-650$ & $\begin{array}{l}\text { Marly-arenaceous } \\
\text { Formation } \\
\text { (sandstone) }\end{array}$ & Slope, top & Northern & $5.0-15.0$ & $82.0-95.0$ & $6.0-6.4$ & $75.3-77.4$ \\
\hline $\begin{array}{l}\text { Aceri obtusati- } \\
\text { Quercetum cerridis } \\
\text { arbutetosum unedonis }\end{array}$ & $400-600$ & $\begin{array}{l}\text { Marly-arenaceous } \\
\text { Formation } \\
\text { (sandstone and marl) }\end{array}$ & Slope & Northern & $13.0-25.0$ & $84.0-90.0$ & $6.2-6.8$ & $43.1-45.8$ \\
\hline $\begin{array}{l}\text { Aceri obtusati- } \\
\text { Quercetum cerridis } \\
\text { Carpinus betulus variant }\end{array}$ & $300-350$ & $\begin{array}{l}\text { Marly alluvial and } \\
\text { colluvial deposits }\end{array}$ & $\begin{array}{l}\text { Impluvium, } \\
\text { alluvial terrace }\end{array}$ & Northern & $1.0-20.0$ & $88.0-95.0$ & $6.8-7.0$ & $39.8-40.6$ \\
\hline $\begin{array}{l}\text { Malo florentinae- } \\
\text { Quercetum frainetto } \\
\text { viburnetosum tini }\end{array}$ & $150-250$ & $\begin{array}{l}\text { Sandy or clayey lake } \\
\text { deposits }\end{array}$ & Slope & Northern & $5.0-13.0$ & $111.3-123.8$ & $5.8-6.1$ & $45.6-51.7$ \\
\hline $\begin{array}{l}\text { Cyclamino hederifolii- } \\
\text { Quercetum ilicis } \\
\text { quercetosum cerridis }\end{array}$ & $400-550$ & $\begin{array}{l}\text { Marly-arenaceous } \\
\text { Formation } \\
\text { (sandstone) }\end{array}$ & Watershed, top & $\begin{array}{l}\text { Northern } \\
\text { (Southern) }\end{array}$ & $15.0-45.0$ & $80.0-96.5$ & $5.4-6.0$ & $51.3-54.7$ \\
\hline $\begin{array}{l}\text { Cyclamino hederifolii- } \\
\text { Quercetum ilicis } \\
\text { cyclaminetosum } \\
\text { hederifolii }\end{array}$ & $400-600$ & $\begin{array}{l}\text { Marly-arenaceous } \\
\text { Formation } \\
\text { (sandstone and marl) }\end{array}$ & Slope, top & $\begin{array}{l}\text { Northern } \\
\text { (Southern) }\end{array}$ & $4.3-21.3$ & $89.5-100.0$ & $7.8-8.2$ & $22.8-25.2$ \\
\hline $\begin{array}{l}\text { Roso sempervirentis- } \\
\text { Quercetum pubescentis } \\
\text { ericetosum multiflorae }\end{array}$ & $350-450$ & Marl & Slope & Southern & $12.5-32.5$ & $91.5-100.5$ & $7.5-7.7$ & $18.7-22.0$ \\
\hline $\begin{array}{l}\text { Roso sempervirentis- } \\
\text { Quercetum pubescentis } \\
\text { quercetosum pubescentis }\end{array}$ & $300-350$ & Marl & Slope & Southern & $25.0-37.5$ & $91.5-108.8$ & $7.9-8.2$ & $18.2-23.7$ \\
\hline
\end{tabular}


Table 11: CCA axes summary statistics and intraset correlations for the environmental variables.

Table 11: Statistika CCA osi in korelacija med okoljskimi spremenljivkami.

\begin{tabular}{lccc}
\hline & Axis 1 & Axis 2 & Axis 3 \\
\hline Eigenvalue & 0.477 & 0.315 & 0.173 \\
Variance in species data & & & \\
$\quad$ Total variance ("inertia”) 4.1484 & & & \\
\% of variance explained & 11.5 & 7.6 & 4.2 \\
$\quad$ Cumulative \% explained & 11.5 & 19.1 & 23.3 \\
$\quad$ Intraset correlations & & & \\
Altitude & 0.059 & 0.968 & 0.161 \\
Aspect & -0.447 & -0.054 & 0.751 \\
Slope & -0.316 & 0.039 & 0.180 \\
Morphology & -0.202 & 0.241 & -0.343 \\
Soil pH & -0.779 & 0.063 & 0.079 \\
Soil sand \% & 0.751 & 0.421 & 0.194 \\
Soil depth & -0.023 & -0.698 & -0.103 \\
\hline
\end{tabular}

Table 12: Hierarchical model of the combination of ecological factors affecting forest landscape diversity. For altitude the whole range of variability was considered; for soil depth and slope angle interquartile ranges were taken into account.

Tabla 12: Hierarhični model kombinacije ekoloških dejavnikov, ki vplivajo na raznolikost gozdne krajine. Za nadmorsko višino so prikazani celotni razponi, za globino tal in naklon pa interkvartilni razpon.

\begin{tabular}{|c|c|c|c|c|c|c|c|c|c|}
\hline $\begin{array}{l}\text { Ecological } \\
\text { factor }\end{array}$ & 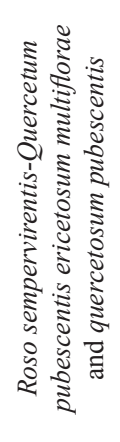 & 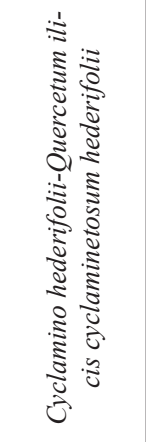 & 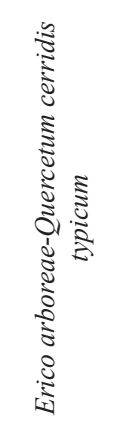 & 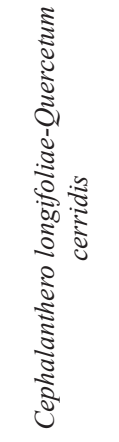 & 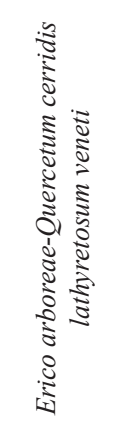 & 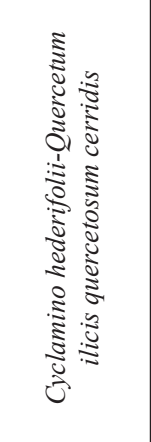 & 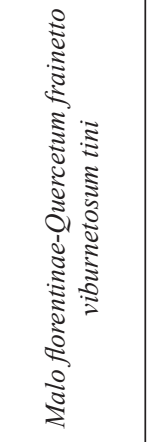 & 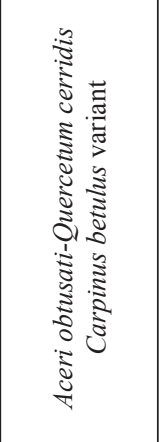 & 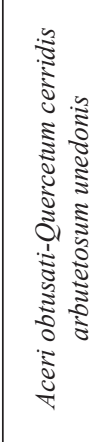 \\
\hline Soil pH & \multicolumn{2}{|c|}{ Subalkaline } & \multicolumn{7}{|c|}{ Neutral to acid } \\
\hline Soil texture & \multicolumn{2}{|c|}{$\begin{array}{c}\text { Silty loam / } \\
\text { Silty clay loam }\end{array}$} & \multicolumn{4}{|c|}{ Loamy sand / Sandy loam / Sandy clay loam } & \multicolumn{3}{|c|}{ Loam / Clay loam } \\
\hline Altitude (m a.s.1.) & \multicolumn{2}{|c|}{$300-600$} & & & \multicolumn{2}{|c|}{$400-550$} & $150-250$ & $300-350$ & $400-600$ \\
\hline Soil depth (cm) & \multicolumn{2}{|c|}{$80-100$} & $<50$ & $80-100$ & $<50$ & $80-100$ & $>100$ & \multicolumn{2}{|c|}{$80-100$} \\
\hline Aspect & Southern & Northern & Southern & Northern & Southern & Northern & \multicolumn{3}{|c|}{ Northern } \\
\hline Morphology & Slopes & Slopes/tops & \multicolumn{2}{|c|}{ Slopes/tops } & Impluvia & $\begin{array}{c}\text { Watersheds/ } \\
\text { tops }\end{array}$ & $\begin{array}{c}\text { Slopes / flat } \\
\text { valley bot- } \\
\text { toms }\end{array}$ & $\begin{array}{c}\text { Impluvia } \\
\text { / alluvial } \\
\text { terraces of } \\
\text { minor water- } \\
\text { courses }\end{array}$ & Slopes \\
\hline Slope angle $\left(^{\circ}\right)$ & $13-38^{\circ}$ & $4-21^{\circ}$ & $5-10^{\circ}$ & $5-15^{\circ}$ & $10-15^{\circ}$ & $15-45^{\circ}$ & $5-13^{\circ}$ & $1-20^{\circ}$ & $13-25^{\circ}$ \\
\hline
\end{tabular}

